\title{
Local Supermassive Black Holes, Relics of Active Galactic Nuclei and the X-ray Background
}

\author{
A. Marconi ${ }^{1 \star}$, G. Risaliti ${ }^{1,2}$, R. Gilli ${ }^{1}$, L. K. Hunt ${ }^{3}$, R. Maiolino ${ }^{1}$, and M. Salvati ${ }^{1}$ \\ ${ }^{1}$ INAF-Osservatorio Astrofisico di Arcetri, Largo Fermi 5, I-50125 Firenze, Italy \\ ${ }^{2}$ Harvard-Smithsonian Center for Astrophysics, 60 Garden street, Cambridge, MA 02138, USA \\ ${ }^{3}$ INAF-Istituto di Radioastronomia-Sez. Firenze, Largo Fermi 5, I-50125 Firenze, Italy
}

Accepted ... . Received ... ; in original form ...

\begin{abstract}
We quantify the importance of mass accretion during AGN phases in the growth of supermassive black holes (BH) by comparing the mass function of black holes in the local universe with that expected from AGN relics, which are black holes grown entirely with mass accretion during AGN phases. The local BH mass function (BHMF) is estimated by applying the well-known correlations between BH mass, bulge luminosity and stellar velocity dispersion to galaxy luminosity and velocity functions. We find that different correlations provide the same BHMF only if they have the same intrinsic dispersion. The density of supermassive black holes in the local universe which we estimate is $\rho_{\mathrm{BH}}=4.6_{-1.4}^{+1.9} h_{0.7}^{2} \times 10^{5} \mathrm{M}_{\odot} \mathrm{Mpc}^{-3}$. The relic BHMF is derived from the continuity equation with the only assumption that AGN activity is due to accretion onto massive BH's and that merging is not important. We find that the relic BHMF at $z=0$ is generated mainly at $z<3$ where the major part of BH's growth takes place. Moreover, the BH growth is anti-hierarchical in the sense that smaller BH's $\left(M_{B H}<10^{7} M_{\odot}\right)$ grow at lower redshifts $(z<1)$ with respect to more massive one's $(z \sim 1-3)$. Unlike previous work, we find that the BHMF of AGN relics is perfectly consistent with the local BHMF indicating the local black holes were mainly grown during AGN activity. This agreement is obtained while satisfying, at the same time, the constraints imposed from the X-ray background. The comparison between the local and relic BHMF's also suggests that the merging process is not important in shaping the relic BHMF, at least at low redshifts $(z<3)$, and allows us to estimate the average radiative efficiency $(\varepsilon)$, the ratio between emitted and Eddington luminosity $(\lambda)$ and the average lifetime of active BH's. Our analysis thus suggests the following scenario: local black holes grew during AGN phases in which accreting matter was converted into radiation with efficiencies $\varepsilon=0.04-0.16$ and emitted at a fraction $\lambda=0.1-1.7$ of the Eddington luminosity. The average total lifetime of these active phases ranges from $\simeq 4.5 \times 10^{8} \mathrm{yr}$ for $M_{B H}<10^{8} M_{\odot}$ to $\simeq 1.5 \times 10^{8} \mathrm{yr}$ for $M_{B H}>10^{9} M_{\odot}$ but can become as large as $\sim 10^{9} \mathrm{yr}$ for the lowest acceptable $\epsilon$ and $\lambda$ values.
\end{abstract}

Key words: black hole physics - galaxies: active - galaxies: evolution - galaxies: nuclei - quasars: general - cosmology: miscellaneous

\section{INTRODUCTION}

Since the discovery of quasars (Schmidt 1963) it has been suggested that Active Galactic Nuclei (AGN) are powered by mass accretion onto a supermassive Black Hole $(\mathrm{BH})$ with mass in the range $10^{6}-10^{10} \mathrm{M}_{\odot}$ (Salpeter 1964; Zel'dovich \& Novikov 1964; Lvnden-Bell 1969). This paradigm combined with the observed evolution of AGNs implies that a significant number of galaxies in the local

* E-mail:marconi@arcetri.astro.it universe should host a supermassive BH (e.g. Soltan 1982; Cavaliere \& Padovani 1989; Chokshi \& Turner 1992).

Supermassive BH's are now detected in $\sim 40$ galaxies through gas and stellar dynamical methods (Kormendv \& Gebhardt 2001; Merritt \& Ferrarese 2001b). Some galaxies are quiescent (e.g. M32, van der Marel et al. 1997; NGC 3250, Barth et al. 2001) and some are mildly or strongly active (e.g. M87, Marconi et al. 1997; Macchetto et al. 1997; Centaurus A, Marconi et al. 2001; Cygnus A, Tadhunter et al. 2003). The mass of the $\mathrm{BH}$ correlates with some properties of the host 
galaxy such as spheroid (bulge) luminosity and mass (Kormendy \& Richstone 1995; Magorrian et al. 1998), light concentration (Graham et al. 2001) and with the central stellar velocity dispersion (Ferrarese \& Merritt 2000; Gebhardt et al. 2000). The latter correlation was thought to be the tightest but Marconi \& Hunt (2003) have recently shown that all the correlations have similar intrinsic dispersion when considering only galaxies with secure BH detections (see also McLure \& Dunlop 2002; Erwin. Graham, \& Caon 2003). Overall, the dispersion is of the order of 0.3 in $\log M_{\mathrm{BH}}$ at a given value of $L_{\mathrm{bul}}, M_{\mathrm{bul}}$ or $\sigma_{\star}$. The existence of any correlations of $\mathrm{BH}$ and host galaxy bulge properties has important implications for theories of galaxy formation in general and bulge formation in particular. Indeed, several attempts at explaining the origin of these correlations and the difficulties/constraints that they pose to galaxy formation models can be found in the literature (e.g. Silk \& Rees 1998; Cattaneo. Haehnelt. \& Rees 1999; Haehnelt \& Kauffmann 2000; Ciotti \& van Albada 2001; Cavaliere \& Vittorini 2002; Adams et al. 2003, and references therein).

To date, all newly found BH's have masses (or upper limits) in agreement with those expected from the above correlations suggesting that all galaxies host a massive $\mathrm{BH}$ in their nuclei. By applying the correlations between $M_{\mathrm{BH}}$ and host galaxy properties it is then possible to estimate the mass function of local BH's or, more simply, their total mass density $\left(\rho_{\mathrm{BH}}\right)$ in the local universe (e.g. Salucci et al. 1999; Marconi \& Salvati 2002; Yu \& Tremaine 2002; Ferrarese 2002; Aller \& Richstone 2002).

It is important to verify if local BH's are exclusively relics of AGN activity or if other mechanisms, such as merging, play a role. Hereafter we will call 'AGN relics', or simply relics, those black holes which grew up from small seeds (1$10^{3} \mathrm{M}_{\odot}$ ) following mass accretion during AGN phases. For instance, an AGN relic of $10^{9} \mathrm{M}_{\odot}$ is different from a $\mathrm{BH}$ of the same mass but which was formed by the merging of many smaller BH's.

A simple comparison between local and relic BH's was performed by Salucci et al. (1999) and Fabian \& Iwasawa (1999) who determined $\rho_{\mathrm{BH}}$ from the observed X-ray background emission. A revised estimate was obtained by Elvis. Risaliti. \& Zamorani (2002) who found $\rho_{\mathrm{BH}}=(7.5-$ $16.8) \times 10^{5}(\epsilon / 0.1)^{-1} \times 10^{5} \mathrm{M}_{\odot} \mathrm{Mpc}^{-3}$ in disagreement with the estimate from local black holes $\left(\rho_{\mathrm{BH}}=(2.5 \pm 0.4) h_{65}^{2} \times\right.$ $10^{5} \mathrm{M}_{\odot} \mathrm{Mpc}^{-3}$, $\mathrm{Yu} \&$ Tremaine 2002; Aller \& Richstone 2002). Elvis. Risaliti. \& Zamorani (2002) thus suggested that, in order to reconcile this discrepancy, massive BH's should have large accretion efficiencies (i.e. larger than the canonically adopted value of $\epsilon=0.1$ ), hence they should be rapidly rotating. A more detailed comparison was performed by Marconi \& Salvati (2002) who found an agreement between the black hole mass functions (hereafter BHMF) of local and relic black holes. Recently, however, Yu \& Tremaine (2002) and Ferrarese (2002) found a disagreement at large masses $\left(M_{\mathrm{BH}} \geqslant 10^{8} \mathrm{M}_{\odot}\right)$ where more AGN relics are expected relative to local BH's. Thus this issue is still much debated.

The relation between AGN relics and local BH's is also being studied in the framework of coeval evolution of $\mathrm{BH}$ and host galaxy. Several physical models have been proposed in which the fueling of the
$\mathrm{BH}$, hence the AGN activity, is triggered by merging events (in the context of the hierarchical structure formation paradigm, see for instance Kauffmann \& Haehnelt 2000; Volonteri. Haardt. \& Madau 2003; Menci et al.|2003; Wvithe \& Loeb 2003; Hatziminaoglou et al. 2003; Haehnelt 2003 ) or is simply directly related to the star formation history of the host galaxy (e.g. Di Matteo et al. 2003; Granato et al. 2003; Haiman, Ciotti, \& Ostriker 2003). The $\mathrm{BH}$ has then a feedback on the host galaxy through the energy released in the AGN phase (e.g. Silk \& Rees 1998; Blandford 1999; Begelman 2003). As a result of this double interaction (galaxy feeding the BH - AGN feedback on the galaxy), these models can in general reproduce both the observed BH-host galaxy correlations and the AGN luminosity functions (e.g. Haehnelt. Nataraian. \& Rees 1998; Monaco. Salucci. \& Danese 2000; Nulsen \& Fabian 2000 and previous references). However, this big effort in modeling cannot uniquely answer the question if local BH's are relics of AGN's, since a wide range of models with many different underlying assumptions cannot be ruled out with the available observational constraints.

The aim of this paper is to investigate the assumption that massive black holes in nearby galaxies are relics of AGN activity by comparing the local BHMF with that of AGN relics. We remark that in this paper we do not build a physical model of the coevolution between central BH and host galaxy but we compare differential and integrated mass densities (local BH's) with differential and integrated energy densities (AGN's), with the only assumption that AGN activity is caused by mass accretion onto the central BH.

We refine the analysis by Marconi \& Salvati (2002) and evaluate the discrepancies found by other authors between local and relic BHMF's. In Section 2 we estimate the local BHMF by applying the known correlations between $M_{\mathrm{BH}}$ and host galaxy properties to the galaxy luminosity and stellar velocity dispersion function. We also check the selfconsistency of the results, and show that different $M_{\mathrm{BH}^{-}}$ host-galaxy-properties relations provide the same BHMF within the uncertainties. In Section 3 we use the continuity equation to estimate the BHMF of AGN relics and in Section 4 we compare local and relic BHMF's, and find that local BH's are consistent with AGN relics. We then show (Sec.55) that the energetic constraints inferred from the $\mathrm{X}$-ray background $(\mathrm{XRB})$ are also satisfied and that there is no discrepancy between $\rho_{\mathrm{BH}}$ of local BH's and that expected from the XRB. In Sections 6 17 and 8] we discuss constraints on the accretion efficiency $(\epsilon)$ and on the Eddington ratio $\left(\lambda=L / L_{\mathrm{Edd}}\right.$, where $L$ is the AGN luminosity and $L_{\text {Edd }}$ is the Eddington luminosity of the active $\mathrm{BH}$ ), and we estimate the accretion history and the average lifetime of massive BH's. We summarize our results and we draw our conclusions in Section [9]

In this paper we adopt the current "standard" cosmological model, with $H_{0}=100 h \mathrm{~km} \mathrm{~s}^{-1} \mathrm{Mpc}^{-1}$ and $h=0.7$, $\Omega_{M}=0.3$ and $\Omega_{\Lambda}=0.7$.

\section{THE MASS FUNCTION OF LOCAL BLACK HOLES}

The first step of the analysis presented here consists in the determination of the mass function of local BH's, i.e. black 
Table 1. Adopted galaxy properties per morphological types.

\begin{tabular}{lllllr}
\hline & E & S0 & Sab & Scd & Notes \\
\hline Morph. Fraction & $0.11 \pm 0.03$ & $0.21 \pm 0.05$ & $0.43 \pm 0.07$ & $0.19 \pm 0.07$ & 1 \\
m(Bulge)-m(Total) & 0 & $0.64 \pm 0.30$ & $1.46 \pm 0.56$ & $2.86 \pm 0.59$ & 2 \\
m(Bulge)-m(Total) & 0 & $0.60 \pm 0.50$ & $1.78 \pm 1.01$ & $2.78 \pm 1.21$ & 3 \\
\hline
\end{tabular}

1. From Fukugita. Hogan. \& Peebles 1998 (see text for details).

2. B band. Estimate by Aller \& Richstone (2002) based on data from Simien \& de Vaucouleurs 1986.

3. $\mathrm{H}$ band. Based on data from Hunt. Pierini. \& Giovanardi 2003.

holes residing in nearby galaxies. The sample of galaxies with dynamically measured BH masses is small $(\sim 40)$ and not selected with well defined criteria. Thus it is useless for a direct determination of the local black hole mass function. However, the BHMF can be derived by applying the existing relations between $M_{\mathrm{BH}}$ and host galaxy properties to galaxy luminosity or velocity functions. After we have described the adopted formalism, we will verify the consistency of the $M_{\mathrm{BH}}-\sigma_{\star}$ and $M_{\mathrm{BH}}-L_{\mathrm{bul}}$ relations in providing the same BHMF within the uncertainties. We will then estimate the BHMF for early and all galaxy types, showing that different galaxy luminosity/velocity functions provide BHMF's which are in agreement within the uncertainties.

\subsection{Formalism}

We describe here the simple formalism which is commonly used to derive the BHMF from galaxy luminosity or velocity functions.

We define $\phi(x) d x$ as the number of galaxies per unit comoving volume with observable $x$ (e.g. luminosity $L$, or stellar velocity dispersion $\sigma$ ) in the range $x, x+d x$. The observable $y$ (e.g. $M_{\mathrm{BH}}$, the $\mathrm{BH}$ mass) is related to $x$ through the $\log$-linear relation $\log y=a+b \log x$ and $\Delta(y)$ is the intrinsic dispersion in $\log y$ at constant $\log x$. Assuming a log-normal distribution then

$P(\log y \mid \log x)=\frac{1}{\sqrt{2 \pi} \Delta_{y}} \exp \left[-\frac{1}{2}\left(\frac{\log y-a-b \log x}{\Delta(y)}\right)^{2}\right]$

where $P(\log y \mid \log x) d \log y$ is the probability that $y$ is in the range $\log y, \log y+d \log y$ for a given $\log x$. Thus the number of galaxies with $x, y$ in the ranges $x, x+d x$ and $y, y+d y$ is

$\Phi(y, x) d x d y=\frac{P(\log y \mid \log x)}{y \ln 10} d y \phi(x) d x$

$\Phi(y) d y$, the number of galaxies with $y$ in the range $y, y+d y$, is thus the convolution of $\phi$ and $P$ :

$\Phi(y)=\int_{0}^{+\infty} \frac{P(\log y \mid \log x)}{y \ln 10} \phi(x) d x$

In the limit of zero-intrinsic dispersion:

$\Phi(y)=\frac{x^{1-b} \phi(x)}{10^{a} b}$

with $\log y=a+b \log x$. After substituting $y$ with $M_{\mathrm{BH}}$ and, for instance, $x$ with $L$, the spheroid luminosity, the total mass density in BH's is simply

$\rho_{\mathrm{BH}}=\int_{0}^{+\infty} M \Phi(M) d M=$

$$
\int_{0}^{+\infty} d M \int_{0}^{+\infty} d L \frac{1}{\ln 10} P(\log M \mid \log L) \phi(L)
$$

and in the zero intrinsic dispersion case:

$\left(\rho_{\mathrm{BH}}\right)_{0}=10^{a} \int_{0}^{+\infty} L^{b} \phi(L) d L$

where $M=M_{\mathrm{BH}}$ for simplicity.

Inverting the order of integration in Eq. 5 and integrating on $M_{\mathrm{BH}}$ one finally gets

$\rho_{\mathrm{BH}}=\mathrm{e}^{\frac{1}{2}\left[\Delta\left(M_{\mathrm{BH}}\right) \ln 10\right]^{2}} \rho_{\mathrm{BH}_{0}}$

A non-null $\Delta\left(M_{\mathrm{BH}}\right)$ increases $\rho_{\mathrm{BH}}$ by a factor $\exp \left[\frac{1}{2}\left(\Delta\left(M_{\mathrm{BH}}\right) \ln 10\right)^{2}\right]$ with respect to the zero dispersion case, a fact already noted by $\mathrm{Yu} \&$ Tremaine (2002).

\subsection{Consistency of the $M_{\mathrm{BH}}-\sigma_{\star}$ and $M_{\mathrm{BH}}-L_{\mathrm{bul}}$ Relations}

Several authors (e.g. Salucci et al. 1999; Marconi \& Salvati 2002; Yu \& Tremaine 2002; Ferrarese 2002; Aller \& Richstone 2002) adopted the method just described to determine the mass function of local BH's. In the most recent works the $M_{\mathrm{BH}}-\sigma_{\star}$ correlation has been preferred to $M_{\mathrm{BH}}-L_{\mathrm{bul}}$ on the ground that it is tighter and, moreover, Yu \& Tremaine (2002) found a factor 2 discrepancy between the values of $\rho_{\mathrm{BH}}$ determined by applying the $M_{\mathrm{BH}}-\sigma_{\star}$ and $M_{\mathrm{BH}}-L_{\mathrm{bul}}$ relations. Thus, we first investigate this inconsistency of the $M_{\mathrm{BH}}-\sigma_{\star}$ and $M_{\mathrm{BH}}-L_{\mathrm{bul}}$ relations by examining the BHMF's derived by applying the two relations to the velocity and luminosity functions obtained from the same sample of galaxies.

We consider the SDSS sample of 9000 early type galaxies from Bernardi et al. 2003a for which the luminosity and velocity functions were determined independently (Bernardi et al. 2003b; Sheth et al. 2003). By 'independently' we mean that the velocity function was derived by directly measuring $\sigma_{\star}$ of all galaxies and not by applying the Faber-Jackson relation (hereafter FJ) to the galaxy luminosity function (e.g. Gonzalez et al. 2000; Aller \& Richstone 2002; Ferrarese 2002). Sheth et al. (2003) compared their velocity function with that derived applying the FJ relation to the luminosity function and found that it is fundamental to take into account the intrinsic dispersion of the relation in order to obtain the correct velocity function. It is consequently expected that the same might apply to the determination of the BHMF.

To derive the BHMF using the $M_{\mathrm{BH}}-\sigma_{\star}$ relation we apply Eq. 3] For the galaxy velocity function $\phi$ we use the 

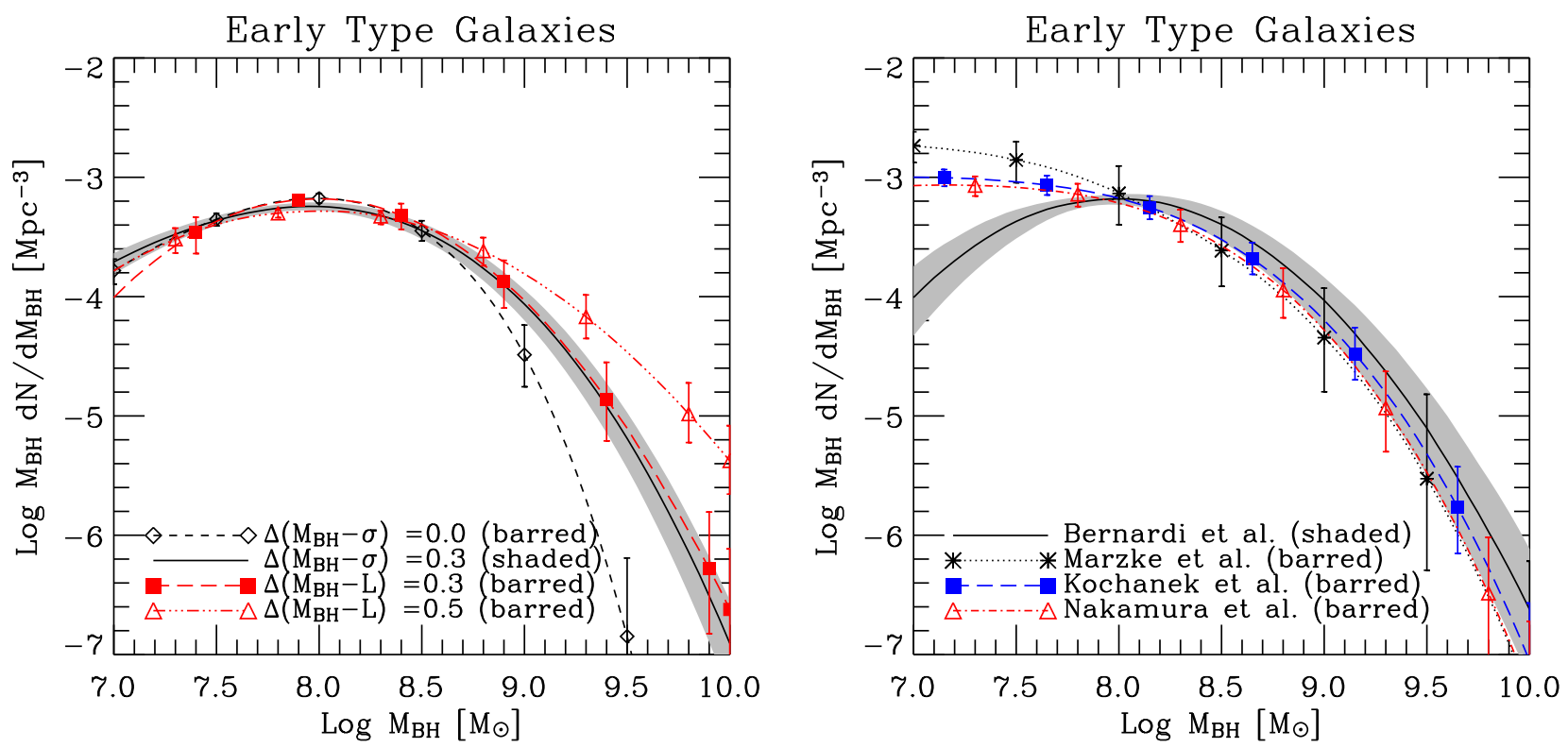

Figure 1. (a) Local BHMF for early type galaxies based on the SDSS sample of Bernardi et al. (2003a). The shaded area and error bars ("barred") indicate the 16 and $84 \%$ percentiles of 1000 Montecarlo realizations of the local BHMF and correspond to $\pm 1 \sigma$ uncertainties (see text). The $\Delta$ 's indicate the assumed intrinsic dispersions of the $M_{\mathrm{BH}}-\sigma_{\star}$ or $M_{\mathrm{BH}}-L_{\mathrm{bul}}$ relations. (b) Local BHMF for early type galaxies derived using the luminosity functions from different surveys (Bernardi et al. 2003b; Marzke et al. 1994; Kochanek et al. 2001; Nakamura et al. 2003) and the $M_{\mathrm{BH}^{-}} L_{\text {bul }}$ relation with $\Delta\left(M_{\mathrm{BH}}\right)=0.3$. The shaded area and errorbars have the same meaning as in (a).

parameterized form for early type galaxies by Sheth et al. (2003). For the $M_{\mathrm{BH}}-\sigma_{\star}$ relation we use the coefficients obtained by fitting the 'Group 1' galaxies of Marconi \& Hunt 2003 (i.e. galaxies with 'secure' BH mass determinations):

$\log M_{\mathrm{BH}}=(8.30 \pm 0.07)+(4.11 \pm 0.33)\left(\log \sigma_{\star}-2.3\right)$

The slope is in agreement with that of Tremaine et al. $2002(4.02 \pm 0.32)$ but there is a larger normalization $\left(8.13 \pm 0.06\right.$ i.e. $\sim 0.2$ in $\left.\log M_{\mathrm{BH}}\right)$. This choice of the coefficients for the $M_{\mathrm{BH}}-\sigma_{\star}$ relation is made for consistency since, in the following, we use the coefficients of the $M_{\mathrm{BH}^{-}}$ $L_{\text {bul }}$ relation determined for the same 'Group 1' galaxies by Marconi \& Hunt (2003). The local BHMF's derived with the $M_{\mathrm{BH}}-\sigma_{\star}$ relation assuming intrinsic dispersions $\Delta\left(M_{\mathrm{BH}}\right)=0$ and $\Delta\left(M_{\mathrm{BH}}\right)=0.30$ are shown in Fig. 11. The shaded area and errorbars indicate the 16 and $84 \%$ percentiles of 1000 Montecarlo realizations of the local BHMF. The realizations were obtained by randomly varying the input parameters assuming that they are normally distributed with $1 \sigma$ uncertainties given by their measurement errors. For the velocity function we have considered only the error on $\phi^{\star}$, the number density at $\sigma^{\star}$, since the other errors are strongly correlated among themselves (Sheth et al. 2003). The 16 and $84 \%$ percentiles indicate the $\pm 1 \sigma$ uncertainties on the logarithm of the local BHMF, whose values from the Montecarlo realizations are normally distributed at a given $M_{\mathrm{BH}}$.

To derive the BHMF using the $M_{\mathrm{BH}}-L_{\text {bul }}$ relation we again apply Eq. 3. The galaxy luminosity function $\phi$ by Bernardi et al. (2003b) is given as a function of the total galaxy light. Since the correlation of $M_{\mathrm{BH}}$ is with bulge light, we need to apply a correction $\Delta m$ in the case of S0 galaxies to transform from total to bulge luminosity. Follow- ing $\mathrm{Yu}$ \& Tremaine (2002), the luminosity function for the bulges of $\mathrm{S} 0$ galaxies is directly given by

$\phi_{b}(m)=\frac{f_{\mathrm{S} 0}}{f_{\mathrm{E}}+f_{\mathrm{S} 0}} \phi(m-\Delta m)$

where $\phi$ is the luminosity function of early type galaxies, $m$ is the absolute bulge magnitude, $\Delta m=m-m_{\text {total }}$ and $f_{\mathrm{E}}$, $f_{\mathrm{S} 0}$ are the fractions of $\mathrm{E}$ and $\mathrm{S} 0$ galaxies with respect to the total galaxy population. The galaxy type fractions and $\Delta m$ used in this paper are shown in Table 1 The morphological type fractions are from Fukugita. Hogan. \& Peebles 1998 (their Sbc fraction has been evenly split between Sab and Scd). The $1 \sigma$ uncertainties are a conservative estimate we made after comparing various determinations of the morphological type fractions available in the literature (see the discussion in Fukugita. Hogan. \& Peebles 1998). The $\Delta m$ for the $B$ band are those estimated by Aller \& Richstone (2002) by rebinning the Simien \& de Vaucouleurs 1986 data in the appropriate bins of galaxy types. The $\Delta m$ for the $H$ band are based on data from Hunt, Pierini, \& Giovanardi 2003 and the values for S0 galaxies were taken from the analysis by Marconi \& Hunt (2003). The $\Delta m$ values are little dependent on the photometrical band at least within the considerable scatter, therefore in the following analysis we will always use the $B$ band data regardless of the photometric band in which $\phi$ was measured. Applying the above corrections to the early-type $r^{\star}$ band luminosity function by Bernardi et al. (2003b) and the color transformation $r^{\star}-K=2.8 \pm 0.2$ we obtain the luminosity function of the bulges of early types in the $K$ band. Cole et al. (2001) estimate $K=z^{\star}-2.12$ which, combined with the average value for the early type galaxies $r^{\star}-z^{\star}=$ 0.68 (Bernardi et al. 2003b), provides the required color. 
Table 2. Mass density of local BH's for early and all galaxy types (Col. 3 and 4 , respectively). Col. 1 indicates the galaxy luminosity or velocity function which was combined with the $M_{\mathrm{BH}}$-host-galaxy-property in Col. 2 to determine the local BHMF. All densities are computed with $\Delta\left(M_{\mathrm{BH}}\right)=0.3$. Uncertainties are the 16 and $84 \%$ percentiles of the Montecarlo realizations described in the text. These correspond to $\pm 1 \sigma$ uncertainties.

\begin{tabular}{lllc}
\hline \hline $\begin{array}{l}\text { Adopted Luminosity } \\
\text { or Velocity Function }\end{array}$ & $\begin{array}{l}M_{\mathrm{BH}}{ }^{-} \\
\text {-host-gal.-prop. }\end{array}$ & $\begin{array}{c}\rho_{\mathrm{BH}}(\mathrm{E}+\mathrm{S} 0) \\
{\left[\times 10^{5} \mathrm{M}_{\odot} \mathrm{Mpc}^{-3}\right]}\end{array}$ \\
\hline Sheth et al. 2003 & $M_{\mathrm{BH}} \sigma_{\star}$ & $3.4_{-0.5}^{+0.6}$ & $5.0_{-1.1}^{+1.7}$ \\
Bernardi et al. 2003 & $M_{\mathrm{BH}} L_{\mathrm{K}, \text { bul }}$ & $3.8_{-1.0}^{+1.2}$ & $\cdots$ \\
Nakamura et al. 2003 & $M_{\mathrm{BH}} L_{\mathrm{K}, \mathrm{bul}}$ & $2.5_{-0.8}^{+1.2}$ & $4.4_{-1.3}^{+2.0}$ \\
Kochanek et al. 2001 & $M_{\mathrm{BH}}-L_{\mathrm{K}, \mathrm{bul}}$ & $3.3_{-0.7}^{+1.0}$ & $4.5_{-1.1}^{+1.4}$ \\
Marzke et al. 1994 & $M_{\mathrm{BH}}-L_{\mathrm{K}, \mathrm{bul}}$ & $3.4_{-1.5}^{+2.7}$ & $4.5_{-1.7}^{+3.1}$ \\
\hline All L- or V- Func. & $\ldots$ & $\ldots$ & $4.6_{-1.4}^{+1.9}$ \\
\hline \hline
\end{tabular}

Fukugita. Shimasaku. \& Ichikawa (1995) estimate $r^{\star}-z^{\star}=$ $0.79: 0.63: 0.70: 0.65: 0.57$ (E:S0:Sab:Sbc:Scd) and this justifies our conservative choice of the scatter which also allows us to apply the same color correction to all morphological types. For the $M_{\mathrm{BH}}-L_{\mathrm{bul}}$ relation we use the coefficients obtained by fitting the 'Group 1' galaxies of Marconi \& Hunt (2003) in the $K$ band:

$$
\log M_{\mathrm{BH}}=(8.21 \pm 0.07)+(1.13 \pm 0.12)\left(\log L_{\mathrm{K}, \mathrm{bul}}-10.9\right)(10)
$$

where $L_{\mathrm{K} \text {,bul }}$ is in units of $\mathrm{L}_{K \odot}$. The local BHMF derived with the $M_{\mathrm{BH}}-L_{\mathrm{bul}}$ relation assuming intrinsic dispersions $\Delta\left(M_{\mathrm{BH}}\right)=0.30$ and $\Delta\left(M_{\mathrm{BH}}\right)=0.5$ are plotted in 1 . As before, the 16 and $84 \%$ percentiles of 1000 Montecarlo realization of the local BHMF indicate $\pm 1 \sigma$ uncertainties (shaded area and errorbars).

As clear from Fig. 17 the effect of including the intrinsic dispersion in the $M_{\mathrm{BH}}-\sigma_{\star}$ and $M_{\mathrm{BH}}-L_{\mathrm{bul}}$ correlations is that of softening the high mass decrease of the BHMF, thus increasing the total density. But the most important result is that in order to provide the same BHMF, the relations $M_{\mathrm{BH}}-\sigma_{\star}$ and $M_{\mathrm{BH}}-L_{\mathrm{bul}}$ must have the same intrinsic dispersion to within 0.1 in log. Yu \& Tremaine (2002) found that $\rho_{\mathrm{BH}}\left[M_{\mathrm{BH}}-L_{\mathrm{bul}}\right] \sim 2 \rho_{\mathrm{BH}}\left[M_{\mathrm{BH}}-\sigma_{\star}\right]$ by using $\Delta\left(M_{\mathrm{BH}}\right)=0$ for $M_{\mathrm{BH}}-\sigma_{\star}$ and $\Delta\left(M_{\mathrm{BH}}\right)=0.5$ for $M_{\mathrm{BH}}-L_{\mathrm{bul}}$. This discrepancy can be entirely ascribed to the effect quantified by Eq. 7 Indeed, the densities $\rho_{\mathrm{BH}}$ of the BHMF's plotted in Fig. 11 are $\rho_{\mathrm{BH}}=2.7_{-0.4}^{+0.5} \times 10^{5} \mathrm{M}_{\odot} \mathrm{Mpc}^{-3}\left[M_{\mathrm{BH}^{-}}\right.$ $\sigma_{\star}$ with $\Delta\left(M_{\mathrm{BH}}\right)=0$, in agreement with $\mathrm{Yu} \&$ Tremaine (2002) $]$ and $\rho_{\mathrm{BH}}=5.5_{-1.3}^{+2.0} \times 10^{5} \mathrm{M}_{\odot} \mathrm{Mpc}^{-3}\left[M_{\mathrm{BH}}-L_{\mathrm{bul}}\right.$ with $\left.\Delta\left(M_{\mathrm{BH}}\right)=0.5\right]$. Conversely, with $\Delta\left(M_{\mathrm{BH}}\right)=0.30$, one obtains $3.4_{-0.5}^{+0.6} \times 10^{5} \mathrm{M}_{\odot} \mathrm{Mpc}^{-3}\left(M_{\mathrm{BH}^{-}} \sigma_{\star}\right)$ and $3.8_{-1.0}^{+1.2} \times$ $10^{5} \mathrm{M}_{\odot} \mathrm{Mpc}^{-3}\left(M_{\mathrm{BH}}-L_{\mathrm{bul}}\right)$ and these two values are in excellent agreement. The densities in massive BH's were evaluated in the $\log \left(M_{\mathrm{BH}} / \mathrm{M}_{\odot}\right)=6-10$ range and the same range will be considered throughout the rest of the paper.

One advantage of using the $M_{\mathrm{BH}}-\sigma_{\star}$ relation is that the uncertainties on the derived BHMF are smaller than in the case of $M_{\mathrm{BH}}-L_{\mathrm{bul}}$. This is because with the $M_{\mathrm{BH}}-\sigma_{\star}$ relation one does not have to apply any correction for the bulge fraction. On the other hand, measuring stellar velocity dispersions is much more difficult than measuring galaxy luminosities; thus it is clear that the two relations $M_{\mathrm{BH}}-\sigma_{\star}$ and $M_{\mathrm{BH}}-L_{\mathrm{bul}}$ should complement each other.

In summary, the use of the same intrinsic dispersion for the $M_{\mathrm{BH}}-\sigma_{\star}$ and $M_{\mathrm{BH}}-L_{\mathrm{bul}}$ relations provides perfectly consistent BHMF's with the same mass densities $\rho_{\mathrm{BH}}$. This is a confirmation of the results by Marconi \& Hunt (2003) who showed that, when considering only secure BH measurements, the $M_{\mathrm{BH}}-\sigma_{\star} M_{\mathrm{BH}}-L_{\mathrm{bul}}$ and $M_{\mathrm{BH}}-M_{\mathrm{bul}}$ relations have similar intrinsic dispersions $(\sim 0.3$ in log). When not taking into account the intrinsic dispersion of the $M_{\mathrm{BH}} \sigma_{\star}$ relation, the local BHMF is systematically underestimated at the high mass end, where the disagreement with the BHMF of AGN relics has been claimed.

\subsection{The Black Hole Mass Function for Early Type Galaxies}

Having established that both $M_{\mathrm{BH}^{-}} \sigma_{\star}$ and $M_{\mathrm{BH}}-L_{\mathrm{bul}}$ relations provide consistent BHMF's we can now evaluate the effects of using luminosity functions from different galaxy surveys and photometric bands in determining the BHMF in early type galaxies.

In Fig. 10 we compare the local BHMF's for early type galaxies obtained from different galaxy luminosity functions, in different photometric bands. We use the luminosity functions by Bernardi et al. (2003b), Marzke et al. (1994), Kochanek et al. (2001), and Nakamura et al. (2003) with details of the derivation specified in the following.

- Bernardi et al. 2003b: this is the same BHMF plotted in panel a derived using the $M_{\mathrm{BH}}-L_{\mathrm{bul}}$ relation.

- Marzke et al. 1994: we use the luminosity functions per morphological type from the CfA survey. The luminosities are in Zwicky magnitudes, $M_{Z}$, and we apply the color transformations directly measured by Kochanek et al. (2001) computing $M_{Z}-K$ for all the objects used for the luminosity functions ( $K$ is obtained from the 2MASS catalogue): $M_{Z}-K=(4.1 \pm 0.65 ; 3.95 \pm 0.65 ; 3.79 \pm 0.56 ; 3.34 \pm 0.64)$ for (E;S0;Sa-Sb;Sc-Sd). The $K$ magnitudes used are isophotal magnitudes $K_{20}$ and the correction to total magnitudes is $K_{t o t}=K_{20}-(0.2 \pm 0.04)$ Kochanek et al. 2001). Then we use the bulge-total correction and the $M_{\mathrm{BH}}-L_{\mathrm{K} \text {,bul }}$ relation from Tab. 1

- Kochanek et al. 2001: we use the luminosity function in the $\mathrm{K}$ band and we apply the correction from $K_{20}$ to $K_{t o t}$ described previously, and we use the morphological fractions and Bulge-Total corrections from Tab. 1

- Nakamura et al. 2003: we use the luminosity function 

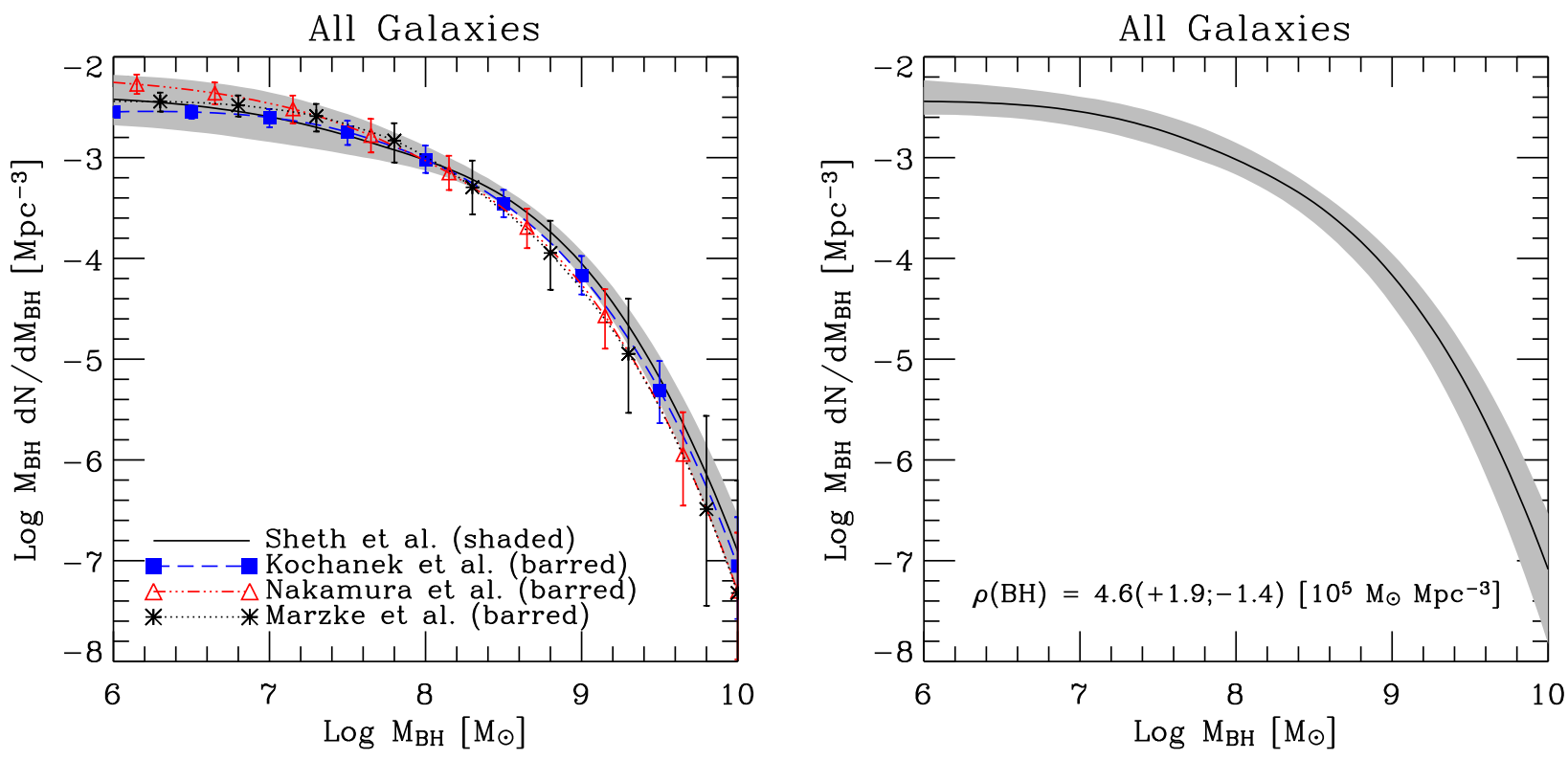

Figure 2. (a) Local BHMF's for all galaxy types derived using different galaxy surveys and shown with the same notation as in Fig. 11 . (b) Best estimate of the local BHMF obtained by combining all the Montecarlo realizations of the BHMF which were used for panel (a). The solid line represents the $50 \%$ percentile and the shaded area is delimited by the 16 and $84 \%$ percentile levels.

in the SDSS $r^{\star}$ band and we apply the color correction $K=$ $r^{\star}-(2.8 \pm 0.2)$ described previously and the Bulge-Total corrections of Tab. 1

As in the previous section, uncertainties are estimated with 1000 Montecarlo realizations of the local BHMF where, in the case of the galaxy luminosity functions, we use only errors on $\Phi^{\star}$, the galaxy number density. All the local BHMF's for early type galaxies are in remarkable agreement within the uncertainties. The discrepancy at the low mass end $\left(M_{\mathrm{BH}}<10^{8} \mathrm{M}_{\odot}\right)$ between the BHMF derived with the Bernardi et al. (2003b) luminosity functions and the others is not significant. It occurs in a region where the luminosity functions of early type galaxies are extrapolated and is due to the different functional forms adopted to fit the data (gaussian for Bernardi et al. (2003b), Schechter functions for the others).

\subsection{The Black Hole Mass Function for All Galaxy Types}

Here we estimate the local BHMF by considering also late morphological types. We use all the luminosity and velocity functions described in the previous section (with the exception of the one by Bernardi et al. (2003b) which is only for early type galaxies). For the velocity function of late type galaxies we take the estimate made by Sheth et al. (2003) and shown in their Fig. 6. When necessary, we apply the bulge-to-total correction with the numbers in Tab. [1 and we then use either the $M_{\mathrm{BH}}-L_{\mathrm{bul}}$ or the $M_{\mathrm{BH}}-\sigma_{\star}$ relation as described in Sec. 2.2

Fig. 22 shows the local BHMF for all galaxies while Tab. 2 reports the estimated $\mathrm{BH}$ mass densities $\rho_{\mathrm{BH}}$ both for early type and all galaxies. These densities are computed for $h=0.7$. All the BHMF's and $\rho_{\mathrm{BH}}$ 's are in agreement within the errors. Finally, in Fig. 20 we present our best estimate of the local BHMF obtained by merging all the random realizations of the BHMF's shown in Fig. 2a and considering the 16, 50 and $84 \%$ percentile levels. Our best estimate of the local density in massive BH's is consequently $\rho_{\mathrm{BH}}=4.6_{-1.4}^{+1.9} \times 10^{5} \mathrm{M}_{\odot} \mathrm{Mpc}^{-3}$. Roughly $70 \%$ of the total $\mathrm{BH}$ density resides in early type galaxies. Our estimate of the density in local BH's is in agreement with Merritt \& Ferrarese (2001a) and with Ferrarese (2002) though, in the latter case, the shape of our BHMF is very different at the high mass end. Our estimate is a factor $\simeq 1.8$ larger than those by Yu \& Tremaine (2002) and Aller \& Richstone (2002) and the reasons for this discrepancy are outlined in Sec. 4

\section{THE MASS FUNCTION OF AGN RELICS}

Once the mass function of local BH's has been determined, the subsequent step is the estimate of the BHMF of AGN relics, i.e. BH's in galactic nuclei which were grown exclusively during active phases from small $\left(1-10^{3} \mathrm{M}_{\odot}\right)$ seeds. We will first describe the continuity equation which will be used to relate the relic BHMF, $N(M, t)$, to the AGN luminosity function $\phi(L, z)$, under the assumption that AGN are powered by mass accretion onto massive BH's. The physical quantity directly related to the mass accretion onto the BH is the total AGN luminosity L. Since AGN luminosity functions are determined in limited energy bands, we will provide suitable bolometric corrections. We will then briefly describe the adopted AGN luminosity functions obtained with our bolometric corrections. Finally we will present our estimates of the relic BHMF's derived from different AGN luminosity functions. 


\subsection{The Continuity Equation}

The Black Hole Mass Function BHMF of AGN relics, $N(M, t)$, can be estimated from the continuity equation (Cavaliere. Morrison. \& Wood 1971; Small \& Blandford 1992) with which, under simple assumptions, it is possible to relate $N(M, t)$ to the AGN luminosity function. If $N(M, t) \mathrm{d} M$ is the comoving number density of BH's with mass in the range $M$ and $M+\mathrm{d} M$ at cosmic time $t$, the continuity equation can be written as:

$$
\frac{\partial N(M, t)}{\partial t}+\frac{\partial}{\partial M}[N(M, t)\langle\dot{M}(M, t)\rangle]=0
$$

where $\langle\dot{M}(M, t)\rangle$ is the "average" accretion rate on the $\mathrm{BH}$ of mass $M$. We adopt the working assumption that AGN's are powered by accretion onto BH's, and that the $\mathrm{BH}$ growth takes place during phases in which the AGN is accreting at a fraction $\lambda$ of the Eddington limit $\left(L=\lambda L_{\mathrm{Edd}}\right)$ converting mass into energy with an efficiency $\epsilon$. We can thus simply relate the AGN Luminosity Function $\phi(L, t)$ $[\phi(L, t) \operatorname{d} \log L$ is the comoving number density of AGNs in the range $\log L, \log L+\mathrm{d} \log L$ at $\operatorname{cosmic}$ time $t]$ to the $\mathrm{BH}$ mass function:

$\phi(L, t) \mathrm{d} \log L=\delta(M, t) N(M, t) \mathrm{d} M$

where $\delta(M, t)$ is the fraction of BH's with mass $M$ which are active at time $t$, i.e. the $\mathrm{BH}$ duty cycle. If a $\mathrm{BH}$ is accreting at a fraction $\lambda$ of the Eddington rate, its emitted luminosity is

$L=\lambda \frac{M c^{2}}{t_{E}}=\epsilon \dot{M}_{\mathrm{acc}} c^{2}$

where $t_{E}$ is the Eddington time, $\epsilon$ is the accretion efficiency and $\dot{M}_{\text {acc }}$ is the matter falling onto the black hole. The growth rate of the $\mathrm{BH}, \dot{M}$, is thus given by $\dot{M}=(1-\epsilon) \dot{M}_{\text {acc }}$, since a fraction $\epsilon$ of the accreted mass is converted into energy and thus escapes the BH. Since $\langle\dot{M}(M, t)\rangle=\delta(M, t) \dot{M}(M, t)$, combining Eqs. 12 and 13 we can write:

$N(M, t)\langle\dot{M}(M, t)\rangle=\frac{(1-\epsilon)}{\epsilon c^{2} \ln 10}[\phi(L, t)]_{L=\lambda \frac{M c^{2}}{t_{E}}} \frac{d L}{d M}$

which can be placed in Eq. 11 where the only unknown function is $N(M, t)$. If $\epsilon$ and $\lambda$ are constant we can then write

$\frac{\partial N(M, t)}{\partial t}=-\frac{(1-\epsilon) \lambda^{2} c^{2}}{\epsilon t_{E}^{2} \ln 10}\left[\frac{\partial \phi(L, t)}{\partial L}\right]_{L=\lambda \frac{M c^{2}}{t_{E}}}$

which can be easily integrated given the AGN luminosity function and the initial conditions.

For initial condition, we assume that, at the starting redshift $z_{s}, \delta\left[M, t\left(z_{s}\right)\right]=1$. This can be interpreted either by saying that at $z_{s}$ all Black Holes are active or that we are following the evolution only of those BH's which were active at $z_{s}$. Thus

$M N\left(M, t_{s}\right)=\left[\phi\left(L, t_{s}\right)\right]_{L=\lambda \frac{M c^{2}}{{ }^{t} E}}$

We will show that the final results are little sensitive to the choice of the initial conditions, provided that $z_{s}>3$, since most of the $\mathrm{BH}$ growth takes place at lower redshifts.

Eq. 15. which represents the continuity equation in the case of constant $\epsilon$ and $\lambda$, can be trivially integrated on $M$ to derive the relation used by various authors
(Padovani. Burg. \& Edelson 1990; Chokshi \& Turner 1992; Yu \& Tremaine 2002; Ferrarese 2002):

$\rho_{\mathrm{BH}}=\frac{1-\epsilon}{\epsilon c^{2}} U_{T}$

note the factor $(1-\epsilon)$ which is needed to account for the part of the accreting matter which is radiated away during the accretion process. $U_{T}$ is the total comoving energy density from AGN's (not to be confused with the total observed energy density) and is given by

$U_{T}=\int_{0}^{z_{s}} d z \frac{d t}{d z} \int_{L_{1}}^{L_{2}} L \phi(L, z) d \log L$

$\phi(L, z) d \log L$ is used if the AGN luminosity function is defined per logarithmic luminosity bin, otherwise it should be $\phi(L, z) d L$.

The right-hand element of the continuity equation, which contains the source function, is null, meaning that we neglect any process which, at time $t$, might 'create' or 'destroy' a BH with mass $M$. Indeed, in the merging process of two BH's, $M_{1}+M_{2} \rightarrow M_{12}$, BH's with $M_{1}$ and $M_{2}$ are destroyed while a $\mathrm{BH}$ with $M_{12}$ is created. We decided to neglect merging of BH's because, at present, the merging rate, $\gamma\left(M_{1}, M_{2}, z\right)$, is very uncertain and dependent on the assumptions of the model with which it is computed. Moreover, the aim of this paper is to assess if local BH's are indeed relics of AGN activity and by neglecting merging we can assess the importance of mass accretion during AGN phases. Yu \& Tremaine (2002) have used an integral version of the continuity equation in order to take into account merging but their approach does not allow a direct comparison of the local and relic BHMF's. Furthermore, the discrepancy they find between local BH's and AGN relics becomes worse if merging is important. Granato et al. (2003) in their physical model of coevolution of $\mathrm{BH}$ and host galaxy find that the growth of the $\mathrm{BH}$ is due to mass accretion. Similarly, Haiman. Ciotti. \& Ostriken (2003) find that merging of BH's is important only at high redshifts while, at lower $z$, $\mathrm{BH}$ growth is dominated by mass accretion. These reasons support our choice of neglecting the merging process. Indeed merging of BH's might well be very important in shaping the BHMF at high redshifts $(z>3-5)$ but, as we will see, the relic BHMF at $z=0$ is very little dependent on its shape and normalization at $z \sim 3$. Moreover, if the relic BHMF at $z=0$ is changed significantly by merging then its remarkable agreement with the local BHMF in the merging-free case would be a mysterious coincidence (see Sec. 4).

\subsection{The Bolometric Corrections}

The total AGN luminosity $L$, which is the physical quantity directly related to the accretion rate, can be estimated from $L_{x}$, the AGN luminosity in the band $x$, by applying a bolometric correction $f_{b o l, x}=L / L_{x}$. In previous works, several authors have used the bolometric corrections of Elvis et al. (1994) which transform the observed flux in a given energy band into the total flux of the AGN spectrum. However, it is well known that the IR radiation is reprocessed from the UV (e.g. Antonucci 1993), thus one should consider the luminosity $L$ given only by the Optical-UV (the so-called 'big blue bump') and X-ray emission. In radio loud AGN's there is an important contribution from synchrotron 

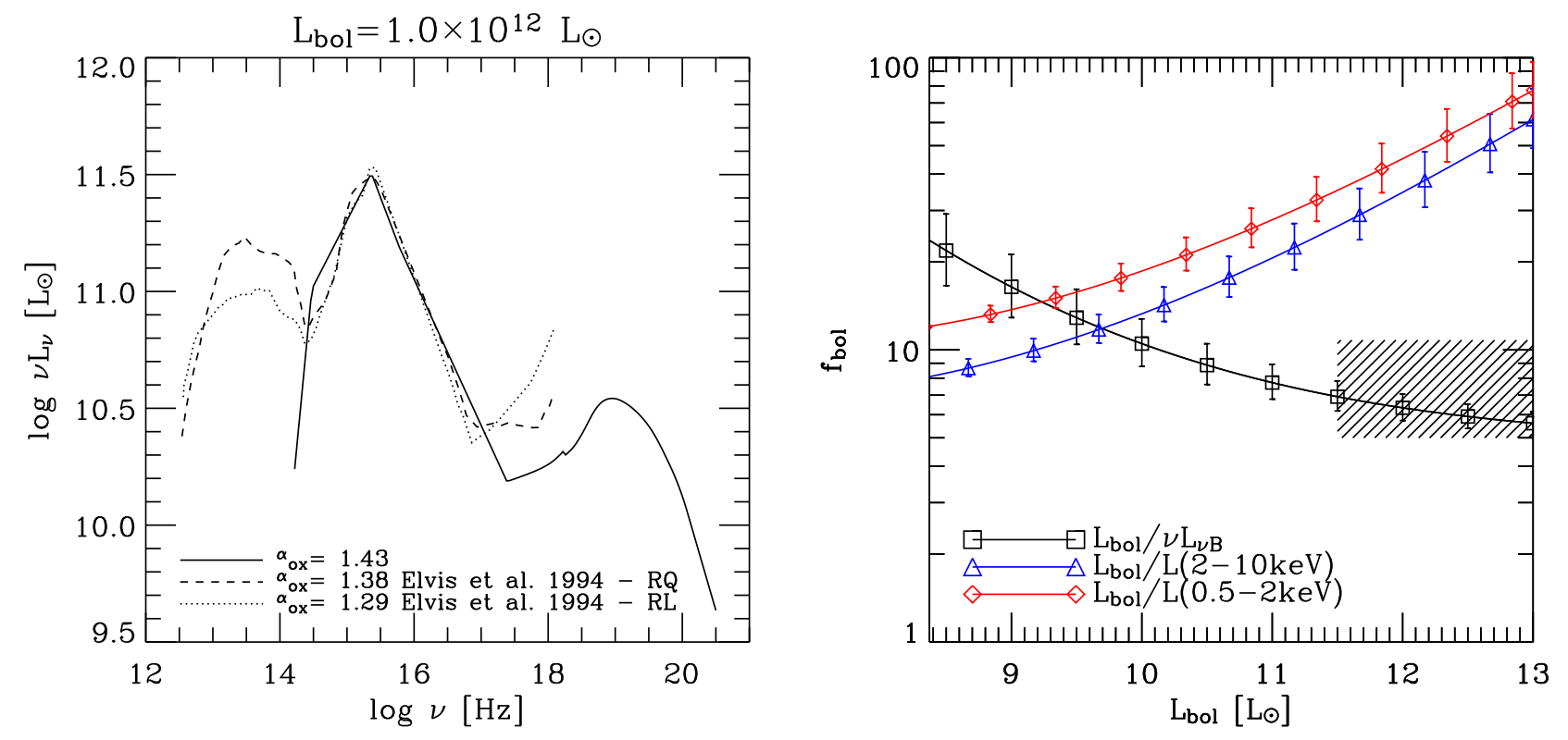

Figure 3. (a) Template spectrum for a $L=10^{12} \mathrm{~L}_{\odot}$ AGN obtained as described in the text (solid line). The dashed and dotted lines represent the radio quiet and radio loud templates by Elvis et al. 1994. (b) Bolometric corrections derived from the spectral templates built as described in the text. The error bars represent the 16 and $84 \%$ percentiles of the 1000 Montecarlo realizations described in the text. The hatched area represents the B band bolometric correction (with $\pm 1 \sigma$ scatter) by Elvis et al. 1994 .

radiation to the IR. However this will not affect our average bolometric corrections since the number of radio loud AGN's is small, $\sim 10 \%$ of the whole AGN population. In the Elvis et al. 1994 spectral templates, the $1 \mu \mathrm{m}-\mathrm{X}$-ray range includes $\simeq 2 / 3$ of the total energy; thus one obtains, for instance, $L / \nu_{B} L_{\nu B}=7.9 \pm 2.9$ instead of the commonly used $L / \nu_{B} L_{\nu B}=11.8 \pm 4.3$. Moreover, the Elvis et al. 1994 quasar templates, obtained from an X-ray selected sample of quasars, are X-ray "bright" (see the discussion in Elvis, Risaliti, \& Zamorani 2002) and thus underestimate the bolometric corrections in the X-ray energy bands. Finally the dependence on luminosity of the bolometric corrections cannot be obtained. For these reasons we will derive new estimates of the bolometric corrections as described in the following.

First, we construct a template spectrum. In the opticalUV region our template consists of a broken power-law with $\alpha=-0.44\left(L_{\nu} \propto \nu^{\alpha}\right)$ in the range $1 \mu \mathrm{m}<\lambda<1300 \AA$ and $\alpha=-1.76$ in the range $1200-500 \AA$ (e.g. Telfer et al. 2002; Vanden Berk et al. 2001). At $\lambda>1 \mu \mathrm{m}$ our big blue bump is truncated assuming $\alpha=2$ as in the RayleighJeans tail of a blackbody. The X-ray spectrum beyond 1 $\mathrm{keV}$ consists of a single power law plus a reflection component. The powerlaw has the typical photon index $\Gamma=1.9$ (e.g. George et al. 1998; Perola et al. 2002) and an exponential cutoff at $E_{c}=500 \mathrm{keV}$. Following Ueda et al. (2003), the reflection component is generated with the pexrav model (Magdziarz \& Zdziarski 1995) in the XSPEC package with a reflection solid angle of $2 \pi$, inclination angle of $\cos i=0.5$ and solar abundances. We then rescale the X-ray spectrum to a given $\alpha_{o x}$ (Zamorani et al. 1981) which is defined as

$\alpha_{o x}=-\frac{\log \left[L_{\nu}(2500 \AA) / L_{\nu}(2 \mathrm{keV})\right]}{\log [\nu(2500 \AA) / \nu(2 \mathrm{keV})]}$ and we finally connect with a simple powerlaw the point at $500 \AA$ with that at $1 \mathrm{keV}$. To account for the dependence of $\alpha_{o x}$ on luminosity, we use the relation by Vignali. Brandt. \& Schneider (2003):

$\alpha_{o x}=-0.11 * \log L_{\nu}(2500 \AA)+1.85$

Finally, we assume that the template spectra, hence the bolometric corrections, are independent of redshift.

The template spectrum for a $L=10^{12} \mathrm{~L} \odot \mathrm{AGN}$ is plotted in Fig. 3 and is compared with the radio quiet and radio loud quasar median spectra by Elvis et al. (1994). Our template is in very good agreement in the optical-UV part but is obviously missing the IR bump, which is due to reprocessed UV radiation and thus not considered here. Our template is fainter in X-rays but this is expected since, as already discussed, the quasar sample by Elvis et al. (1994) is X-ray bright.

In Fig. 31] we plot the bolometric corrections $L / \nu_{B} L_{\nu B}$, $L / L(0.5-2 \mathrm{keV})$ and $L / L(2-10 \mathrm{keV})$ derived from the above templates. The errorbars lines represent the 16 and $84 \%$ percentiles from 1000 Montecarlo realization of the spectral templates where we have assumed the following $1 \sigma$ uncertainties for the input parameters: \pm 0.1 for the spectral slopes ( $\alpha$; Telfer et al. 2002; Vanden Berk et al. 2001) in the $1 \mu \mathrm{m}$ $500 \AA$ range and \pm 0.05 for the $\alpha_{o x}$, constant at all luminosities. The latter is a conservative assumption (see, e.g., Yuan et al. 1998) but is made to account for possible, but unaccounted for, systematic errors. As in the case of the local BHMF, the 16 and $84 \%$ percentiles correspond to $\pm 1 \sigma$ uncertainties on the logarithm of the bolometric corrections. The $50 \%$ percentiles can be fit with a 3rd degree polynomial to obtain the following convenient relations:

$\log (L / L(2-10 \mathrm{keV}))=1.54+0.24 \mathcal{L}+0.012 \mathcal{L}^{2}-0.0015 \mathcal{L}^{3}$ 

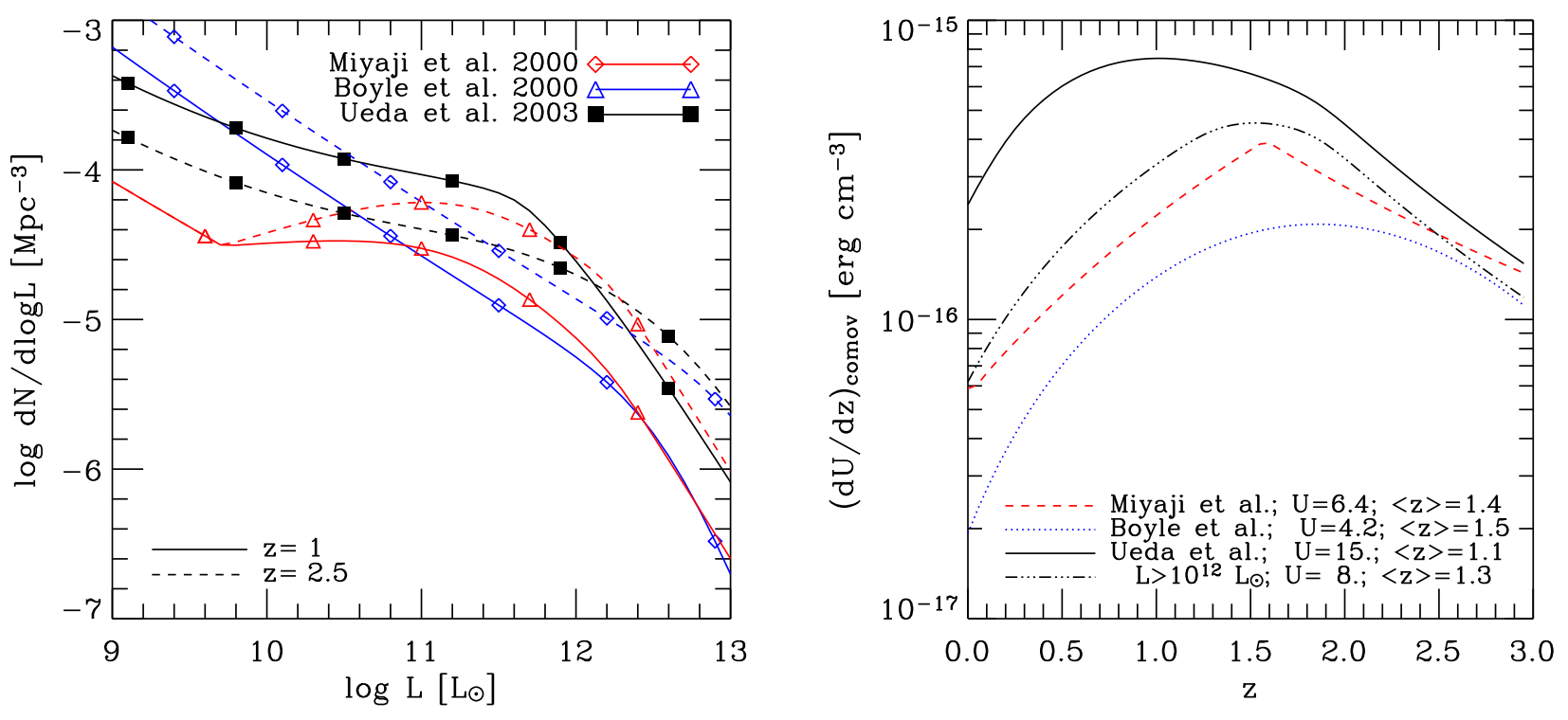

Figure 4. (a) AGN LF's at selected redshifts obtained with the bolometric corrections described in the text. Different symbols indicate different AGN luminosity functions, while different line styles distinguish between redshifts. (b) Differential comoving energy densities of AGN's from different surveys obtained with the bolometric corrections described in the text. In the case of the Ueda et al. (2003) luminosity function we also plot the comoving energy density for high luminosity objects $\left(L>10^{12} \mathrm{~L}_{\odot}\right)$. ' $\mathrm{U}$ ', the total comoving energy density in the redshift range $z=0-3$, is in units of $10^{-16} \mathrm{erg} \mathrm{cm}^{-3}$ and ' $\langle z\rangle$ ' is the average redshift computed following Eq. 228

$\log (L / L(0.5-2 \mathrm{keV}))=1.65+0.22 \mathcal{L}+0.012 \mathcal{L}^{2}-0.0015 \mathcal{L}^{3}$ $\log \left(L / \nu_{B} L_{\nu B}\right)=0.80-0.067 \mathcal{L}+0.017 \mathcal{L}^{2}-0.0023 \mathcal{L}^{3}$

where $\mathcal{L}=(\log L-12)$ and $L$ is the bolometric luminosity in units of $\mathrm{L}_{\odot}$. Hence, the bolometric corrections have lognormal distributions with average values given by the above equations and $\pm 1 \sigma$ scatters (at fixed $L$ ) which can be derived from Fig. 3b. Scatters are given by $\sim 0.05$ for the B band and $\sim 0.1$ for the X-rays, taken independent of $L$ for simplicity. It is worth noting that the B band bolometric correction is in agreement with that by Elvis et al. (1994) whose average value and scatter are shown by the hatched area in Fig. 3 . The scatter of the Elvis et al. (1994) bolometric correction is the standard deviation of their quasar sample while ours are uncertainties on the average values. The average $\alpha_{o x}$ in the $\log \left(L / \mathrm{L}_{\odot}\right)=11.5-12.5$ range is 1.43 , the same value estimated by Elvis. Risaliti. \& Zamorani (2002) after correcting for the biases due to X-ray or optical selection of the parent quasar samples.

\subsection{The Luminosity Function of Active Galactic Nuclei}

In order to ensure a consistent treatment, the AGN luminosity function $\phi(L, t)$ used in the continuity equation must describe the evolution of the entire AGN population. The luminosity $L$ is the total luminosity radiated from the accreting mass and is estimated with the bolometric corrections described in the previous section.

The AGN luminosity functions available in the literature do not describe the entire AGN population because they are the result of surveys performed in "narrow" spectral bands, with given flux limits and selection criteria. For example, the luminosity function of Bovle et al. (2000) includes only quasars selected for their blue color but it is now known there is a population of "red" quasars which is missed. The luminosity function of soft X-ray (0.5-2 $\mathrm{keV}$ ) selected AGNs of Mivaii. Hasinger. \& Schmidt (2000) misses most of the sources with significant X-ray absorption $\left(N_{H}>10^{22} \mathrm{~cm}^{-2}\right)$; it includes mostly broad lined AGNs ( $\sim 80 \%$ of the total) but it is known that, at least locally, there is a dominant population of obscured AGN's (e.g. Maiolino \& Rieke 1995). Current hard X-rays surveys (2-10 keV) are less sensitive to obscuration than optical and soft X-ray ones, thus the very recent luminosity function by Ueda et al. (2003) probes by far the largest fraction of the whole AGN population. However it is restricted only to Compton-thin AGNs $\left(N_{H}<10^{24} \mathrm{~cm}^{-2}\right)$, while it is known that, at least locally, there is a significant fraction of Compton-thick objects (e.g. Risaliti, Maiolino, \& Salvati 1999). In summary, when using the luminosity functions available in the literature, one should know that they describe part of the AGN population, and thus account only for a fraction of the local BHMF.

In this paper, we consider the AGN luminosity functions by Bovle et al. 2000 (band B), Mivaii. Hasinger. \& Schmidt $2000(0.5-2 \mathrm{keV})$ and Ueda et al. $2003(2-10 \mathrm{keV})$. Thus, the AGN luminosity function which will be used in the continuity equation is given by

$\phi(L) d L=\phi\left(L_{x}\right) d L_{x}$

where $x$ is either the $B$, the $0.5-2 \mathrm{keV}$ or the $2-10 \mathrm{keV}$ band and $L=f_{b o l, x} L_{x}$.

The AGN luminosity functions obtained with the bolometric corrections described in the previous section are compared in Fig. 4 at selected redshifts. 

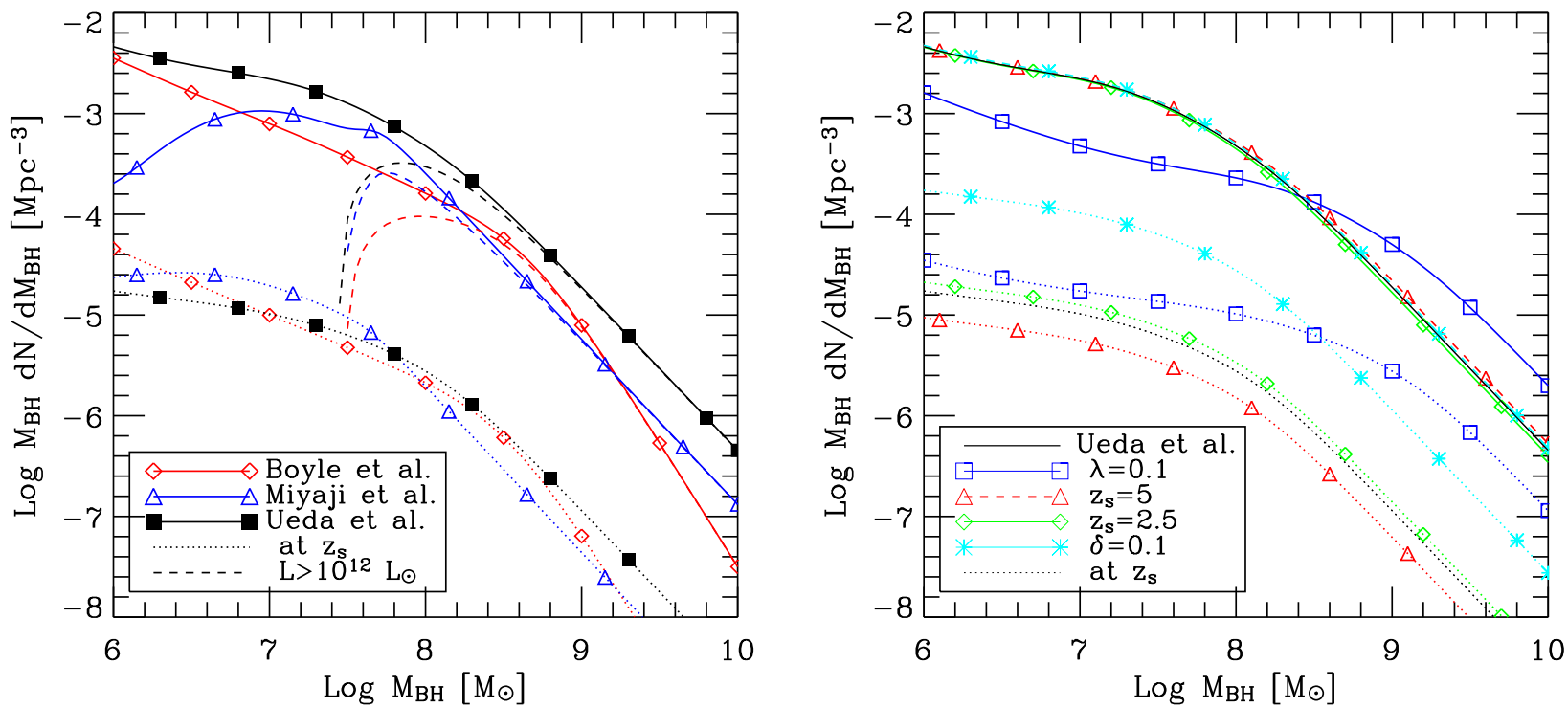

Figure 5. (a) Relic BHMF's at $z=0$ (solid lines) calculated assuming $\epsilon=0.1$ and $\lambda=1$ compared with the relic BHMF's at $z_{s}=3$ (dotted lines). Different symbols indicate the different AGN luminosity functions used. (b) Relic BHMF's obtained from the Ueda et al. (2003) AGN luminosity function but with different $\lambda, z_{s}$ and $\delta$ with respect to panel (a). For comparison, the thick line with no symbols indicates the relic BHMF from Ueda et al. 2003 plotted in (a). As in the previous panels, solid lines are the relic BHMF's at $z=0$, while dotted lines are those at $z_{s}$.

The luminosity functions by Bovle et al. (2000) and Mivaii. Hasinger. \& Schmidt (2000) are in rough agreement at the high $L$ end, meaning that they may be sampling the same quasar population. Indeed most of the objects in the Mivaii. Hasinger. \& Schmidt (2000) sample are broad-lined AGN which, at high $L$, become the quasars observed by Bovle et al. (2000). The disagreement at low luminosities is because the Bovle et al. 2000 luminosity function is extrapolated for $M_{B}>-23$ i.e. for $L<10^{12} \mathrm{~L} \odot$. In contrast the luminosity function by Ueda et al. (2003) samples a larger fraction of the AGN population at all luminosities.

In Fig. 4b we compare the differential comoving energy densities computed in the luminosity ranges $41.5<$ $\log \left(L_{X} / \operatorname{erg} \S^{-1}\right)<48(0.5-2 \mathrm{keV}$ and $2-10 \mathrm{keV}$ bands $)$ and $-28<M_{B}<-21$ ( $B$ band). For the Ueda et al. (2003) luminosity function we also plot the differential comoving energy density for objects with $L>10^{12} \mathrm{~L}_{\odot}$. High luminosity objects provide $\sim 50 \%$ of the total energy density in the Ueda et al. (2003) luminosity function and their emission has a redshift distribution similar to that of the AGN's by Mivaii. Hasinger, \& Schmidt (2000) and Bovle et al. (2000). Clearly, lower luminosity objects contribute significantly at $z<1.5$ and this is an important result of the recent Chandra and XMM surveys (e.g. Hasinger 2003; Fiore et al. 2003; Ueda et al. 2003). The total comoving energy densities in the redshift range $z=0-3$ (i.e. the integrals of the quantities plotted in the figure) are $4.2 \times 10^{-16}$, $6.4 \times 10^{-16}$ and $1.5 \times 10^{-15} \mathrm{erg} \mathrm{cm}^{-3}$, for Bovle et al. 2000, Mivaii. Hasinger. \& Schmidt 2000, and Ueda et al. 2003, respectively. The high $L$ objects in the Ueda et al. (2003) luminosity function provide $8 \times 10^{-16} \mathrm{erg} \mathrm{cm}^{-3}$, i.e. $\sim 50 \%$ of the total energy density.

By applying Eq.17 the mass densities in BH's expected from the above surveys are respectively

$$
\begin{aligned}
\rho_{\mathrm{BH}} & =0.6\left[\frac{1-\epsilon}{9 \epsilon}\right] \times 10^{5} \mathrm{M}_{\odot} \mathrm{Mpc}^{-3} \\
& =0.9\left[\frac{1-\epsilon}{9 \epsilon}\right] \times 10^{5} \mathrm{M}_{\odot} \mathrm{Mpc}^{-3} \\
& =2.2\left[\frac{1-\epsilon}{9 \epsilon}\right] \times 10^{5} \mathrm{M}_{\odot} \mathrm{Mpc}^{-3} \\
& =1.2\left[\frac{1-\epsilon}{9 \epsilon}\right] \times 10^{5} \mathrm{M}_{\odot} \mathrm{Mpc}^{-3}
\end{aligned}
$$

The value derived from the Ueda et al. (2003) luminosity function $\left(2.2 \times 10^{5} \mathrm{M}_{\odot} \mathrm{Mpc}^{-3}\right)$ is already in marginal agreement with $\rho_{\mathrm{BH}}=4.5_{-1.4}^{+1.8} \times 10^{5} \mathrm{M}_{\odot} \mathrm{Mpc}^{-3}$, i.e. the estimate of the local $\mathrm{BH}$ density given in Sec. 2.4

\subsection{The Relics of Active Galactic Nuclei}

Given the AGN luminosity functions (Bovle et al. 2000; Mivaii. Hasinger, \& Schmidt 2000; Ueda et al. 2003) we can integrate the continuity equation assuming that $\lambda=1$ (AGN's emitting at the Eddington luminosity), $\epsilon=0.1$ and $z_{s}=3$. Though we know that the above luminosity functions do not describe the whole AGN population, initially we do not apply any correction for the objects which have been missed.

The relic $\mathrm{BH}$ mass functions are shown in Fig. 57 . As expected, the AGN's traced by the Ueda et al. (2003) luminosity function leave more relics than those traced by the luminosity functions of Bovle et al. (2000) and Mivaii. Hasinger. \& Schmidt (2000). It is also notable that the relic BHMF's at $z=0$ (solid lines) are $\sim 2$ order of magnitude larger than those at $z_{s}=3$ (dotted lines) meaning that most of the $\mathrm{BH}$ growth took place for $z<z_{s}=3$. 
The dashed lines represent the relic BHMF obtained by considering only AGN's with $L>10^{12} \mathrm{~L} \odot$. [In practice this was obtained by multiplying the AGN luminosity functions by $\exp \left(-10^{12} / L\right)$.] The comparison between the dashed and solid lines indicate that today high mass $\mathrm{BH}$ 's $\left(M_{\mathrm{BH}}>\right.$ $\left.10^{8} \mathrm{M}_{\odot}\right)$ grew during quasar phases $\left(L>10^{12} \mathrm{~L}_{\odot}\right)$.

The BHMF's in Fig. 5 a were estimated assuming $\lambda=1$ and $\epsilon=0.1$. It can be seen from Eq. 15 that the efficiency $\epsilon$ is a simple scaling factor and an increase in $\epsilon$ will decrease the level of the relic BHMF. However a variation of the Eddington fraction $\lambda$ will not have a simple scaling effect but it will produce a combination of scaling and translation along the $M$ axis since $\lambda$ also enters the $L=\lambda M c^{2} / t_{E}$ relation. In Fig. 5 p the same relic BHMF derived using Ueda et al. 2003 (thick line with no symbols) is compared with the BHMF obtained assuming $\lambda=0.1$ (line with empty squares). As previously, solid and dotted lines indicate the BHMF at $z=0$ and at $z=z_{s}$ respectively. Decreasing $\lambda$ has the net effect of increasing the number of BH's at high masses $\left(M>10^{8.5} \mathrm{M}_{\odot}\right)$ while decreasing that at lower masses. In the same figure, we also show the BHMF's obtained by assuming that the starting redshift of integration is $z_{s}=5$ (line with empty triangles) and $z_{s}=2.5$ (line with empty diamonds) and that the fraction of active BH's at $z_{0}$ is $\delta=0.1$ instead of 1 (line with stars). The relic BHMF at $z=0$ is almost independent of the initial conditions. Changing the starting assumption $\left(z_{s}\right.$, or $\left.\delta\left[z_{s}\right]\right)$ does not have any appreciable effect provided that $z_{s} \gtrsim 2.5$. This is a consequence of the fact that the main $\mathrm{BH}$ growth takes place at $z<3$ when there is more time available $(\sim 85 \%$ of the age of the universe). At larger redshifts there is too little time for the $\mathrm{BH}$ growth.

In summary, relic BH's grew mainly at low redshifts $(z<3)$ and high mass BH's were produced during quasar activity. The initial conditions for the continuity equation influence little the relic BHMF at $z=0$.

\section{LOCAL BH'S AND AGN RELICS}

In this section we compare the local and relic BHMF's. At first we will consider the relic BHMF's derived in the previous section, without trying to account for the AGN population missing in the adopted luminosity functions. After showing that, as expected, the Ueda et al. (2003) luminosity function encompasses the largest fraction of the AGN population, thus providing a better match to the local BHMF, we will focus on it and try to account for the missing AGN's in a way which also satisfies the constraints imposed by the X-ray background.

In Fig. 6a we compare the local BHMF derived in Sec. 2.4 (Fig. 2p) with the relic BHMF's obtained adopting the Bovle et al. (2000), Mivaii. Hasinger. \& Schmidt (2000), and Ueda et al. (2003) AGN luminosity functions $(\epsilon=0.1$, $\lambda=1$ ) without any correction for the missing AGN population. As shown in the previous section these relic BHMF's are insensitive to the adopted initial conditions thus the parameters they depend on are only $\epsilon$ and $\lambda$.

The first result which can be evinced from the figure is that there is no discrepancy at high masses. All the relic BHMF's are slightly smaller than the local BHMF. As explained previously, this is not significant because all the adopted luminosity functions are missing part of the AGN population. Conversely $\mathrm{Yu} \&$ Tremaine (2002) and Ferrarese (2002) using the Bovle et al. (2000) luminosity function found a significant discrepancy because their relic BHMF at high masses is larger than the local one. The reasons for the solution of this discrepancy are the following.

- We have taken into account the intrinsic dispersion of the $M_{\mathrm{BH}}-\sigma_{\star}$ and $M_{\mathrm{BH}}-L_{\text {bul }}$ correlations thus softening the high mass decrease of the local BHMF.

- We have adopted zero points in the $M_{\mathrm{BH}}-\sigma_{\star}$ and $M_{\mathrm{BH}}$ $L_{\text {bul }}$ correlations which are a factor $\sim 1.6(\sim 0.2$ in $\log )$ larger than those used by $\mathrm{Yu} \&$ Tremaine (2002). These zero points (and slopes) were derived from the analysis of Marconi \& Hunt (2003) and their larger value is a consequence of the rejection of galaxies with unreliable $\mathrm{BH}$ masses.

- We have adopted bolometric corrections which, on average, are $\sim 2 / 3$ of those adopted by previous authors. This is because we have not taken into account the IR emission in the estimate of the bolometric luminosity. The IR bump is produced by reprocessed UV radiation and thus its use in the determination of $L$ would result in overestimated accretion rates.

We now focus on the Ueda et al. (2003) AGN luminosity function since we have established that, as expected, it is the one which provides the most complete description of the AGN population. The aim is to assess whether the relic BHMF can account for the whole local BHMF, i.e. if local BH's can be entirely explained as being AGN relics.

The Ueda et al. (2003) luminosity function only describes the population of Compton-thin AGN's, i.e. objects with absorbing column densities $\log N_{H}<24\left[\mathrm{~cm}^{-2}\right]$. To estimate the correction for the missing Compton-thick sources, we follow Ueda et al. (2003) and assume that there are as many AGN's in the 24-25 bin of $\log N_{H}$ as in the 23-24 one, as indicated by the $N_{H}$ distribution of Risaliti. Maiolino. \& Salvati 1999. Thus, with the $N_{H}$ distribution estimated by Ueda et al. (2003), the luminosity function has to be multiplied by 1.3 in order to account for the Compton-thick AGN's. In reality, the correcting factor is luminosity dependent but, since it is limited to the $1.2-1.4$ range, we chose an average value for simplicity. With this contribution from Compton-thick sources, Ueda et al. (2003) are able to provide a reasonable fit of the $\mathrm{X}$-ray background spectrum. (We will return to this issue in the following section.) While AGN's at $\log N_{H}>25$ are not important contributors of the X-ray background, they do contribute to the relic BHMF. Thus, we assume that for $\log N_{H}>25$ there are as many AGN's as in the 2324 or $24-25 \log N_{H}$ bins. This assumption is also justified by the Risaliti. Maiolino. \& Salvati (1999) $N_{H}$ distribution. The correcting factor becomes then 1.6. Therefore, to compensate for the missing obscured AGN's we apply a correcting factor of 1.6 independently of luminosity. In Fig. 6b the corrected Ueda et al. (2003) luminosity function is used to determine the relic BHMF. We also show the uncertainties ( 16 and $84 \%$ percentiles, i.e. $\pm 1 \sigma$ errors on the log of the relic BHMF as previously) estimated with the usual 1000 Montecarlo realizations of the relic BHMF. These were obtained by varying the number density of the luminosity function ( $\pm 30 \% 1 \sigma$ error on the number density to avoid correlated 

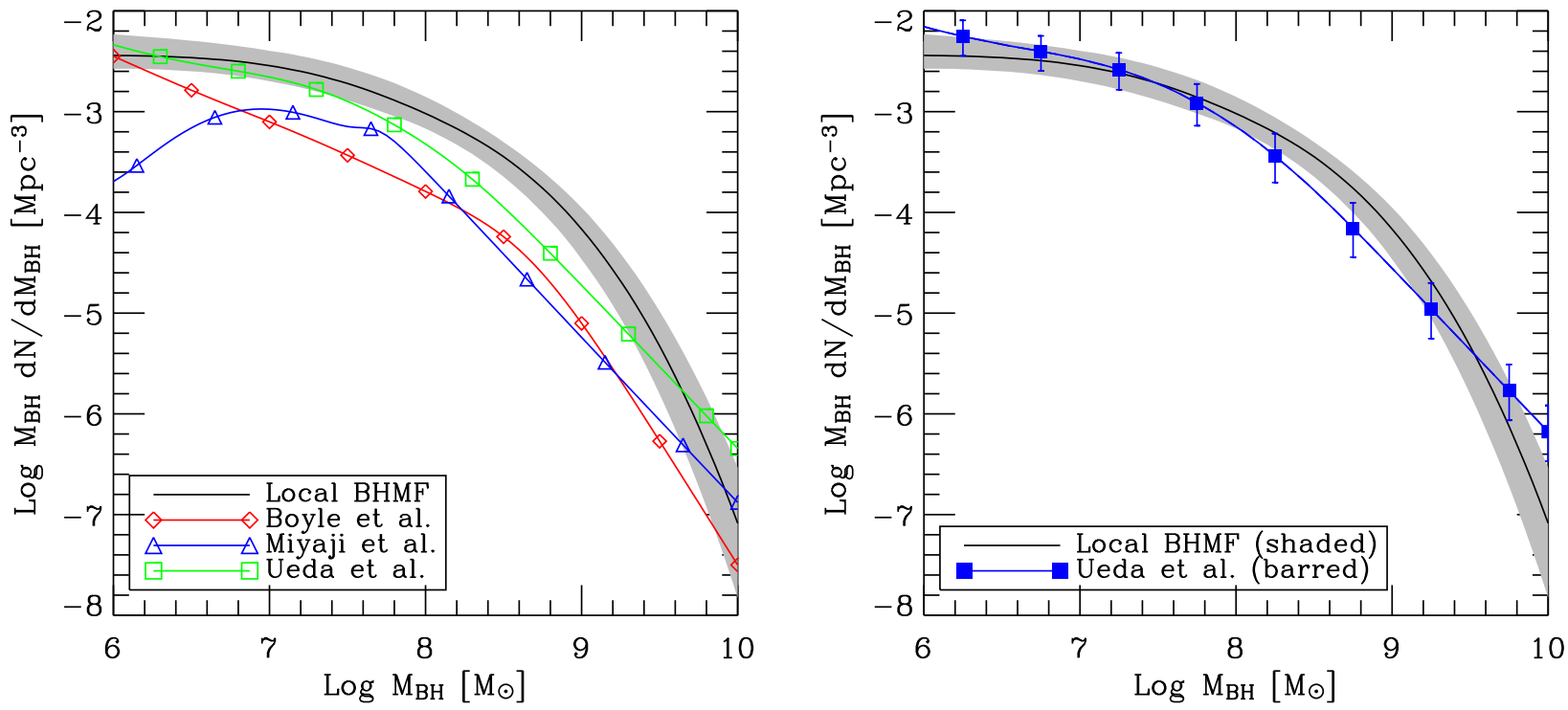

Figure 6. (a) Local and relic BHMF's. The shaded area represents the $\pm 1 \sigma$ uncertainty on the logarithm of local BHMF (see Fig. 20 ). (b) Relic BHMF obtained using the Ueda et al. (2003) hard X-ray luminosity function and accounting for the missing Compton Thick AGN's (see text). The errorbars are the 16 and $84 \%$ percentile levels of 1000 realizations of the relic BHMF (see text). The local BHMF is plotted with the same notation as in the panel (a).

errors on the other parameters), the hard X-ray bolometric correction $\left( \pm 0.11 \sigma\right.$ error on $\left.\log f_{b o l, X}\right)$, and the factor used to correct for the missing Compton-thick AGN's $( \pm 0.31 \sigma$ error).

The relic and local BHMF agree well within the uncertainties. Thus, adopting the best possible description of the whole AGN population, the mass function of relic BH's is in excellent agreement with the mass function of local BH's. Local BH's are thus AGN relics and were mainly grown during active phases in the life of the host galaxy. This agreement has been obtained with $\epsilon=0.1$ and $\lambda=1$ indicating that (i) efficiencies higher than commonly adopted for AGN's are not required, and (ii) the main growth of BH's occurs in phases during which the AGN is emitting close to the Eddington limit. In Section [6] we will explore the locus in the $\lambda-\epsilon$ plane which is permitted by the comparison from the local and relic BHMF's. As discussed in Section 3.1] we have neglected merging in our estimate of the relic BHMF. However, the good agreement between the local and relic BHMF's suggests that the merging process does not significantly affect the build-up of the BHMF, at least in the $z<3$ redshift range.

\section{CONSTRAINTS FROM THE X-RAY BACKGROUND}

From the X-ray background (XRB) light it is possible to estimate the expected mass density of relic BH's (Salucci et al. 1999; Fabian \& Iwasawa 1999; Elvis. Risaliti. \& Zamorani 2002) which can then be compared with the mass density of local BH's. From this comparison, Elvis, Risaliti, \& Zamorani (2002) inferred that massive BH's must be rapidly rotating for the high efficiency needed $(\epsilon>0.15)$ to match the XRB and local $\mathrm{BH}$ mass densities. We will show that, due to the redshift distribution inferred from the new X-ray surveys, the match between the XRB and local $\mathrm{BH}$ mass densities can be obtained without requiring large efficiencies $(\epsilon>0.1$; see also Fabian 2003; Comastri 2003).

The XRB provides another type of constraint. It has been shown (Setti \& Woltier 1989; Comastri et al. 1995; Gilli. Risaliti, \& Salvati 1999) that the XRB spectrum can be reproduced by summing the spectra of the whole AGN population after suitable corrections to take into account the absorption along the line of sight. The AGN population and its redshift distribution is derived from AGN luminosity functions but, in order to fit the XRB spectrum, one has to include a correction for the missing (obscured) AGN's. This correction is the same one which must be adopted here to determine the relic BHMF from the whole AGN population. Thus, the ratio $R_{12}$ between AGN's included and missed in the adopted luminosity functions is a parameter which determines both the relic BHMF and the XRB spectrum (and the X-ray source counts). When rescaling the AGN luminosity function with the $\left(1+R_{12}\right)$ factor to match the local BHMF, one should also verify that it is also possible to match the XRB spectrum and source counts at the same time. In practice, one can use the $R_{12}$ value required by the XRB synthesis models in order to fit the XRB spectrum and source counts. It is beyond the scope of this paper to produce a model synthesis of the XRB but we will show that with $R_{12}$ values found in the literature we can match the local and relic BHMF, and also satisfy the constraints imposed by the X-ray background.

The local density in massive BH's expected from the 
observed X-ray background (XRB) light can be estimated with the relation

$\rho_{\mathrm{BH}}=\frac{1-\epsilon}{\epsilon c^{2}}(1+\langle z\rangle) U_{T}^{\star}$

where $U_{T}^{\star}$ is the total observed (as opposed to comoving) AGN energy density and $\langle z\rangle$ is the average source redshift. The factor $(1-\epsilon)$ is needed to take into account the fact that not all the accreting mass falls into the $\mathrm{BH}$. $U_{T}^{\star}$ can be estimated from the observed $\mathrm{X}$-ray background light as

$U_{T}^{\star}=f_{b o l, X} \times f_{o b s c} \times \frac{4 \pi}{c} I_{X}$

where $I_{X}$ is the total observed surface brightness of the X-ray background (i.e. the integral of the XRB spectrum), $f_{\text {obsc }}$ is the correction to take into account source obscuration in the X-rays (i.e. $f_{\text {obsc }} \times I_{X}$ would be the total XRB surface brightness if AGN's were not obscured) and $f_{b o l, X}$ is the X-ray bolometric correction (see for more details Fabian \& Iwasawa 1999; Salucci et al.|1999; Elvis. Risaliti. \& Zamorani 2002). Elvis. Risaliti. \& Zamorani (2002) estimate $U_{T}^{\star} \sim(1.5-$ 3.4) $\times 10^{-15} \mathrm{erg} \mathrm{cm}^{-3}$.

We first verify that the above formula is consistent with the scheme followed in this paper, and then establish how $\langle z\rangle$ must be computed. The observed background surface brightness at energy $E$ is

$$
\begin{aligned}
& I(E)=\frac{1}{4 \pi} \times \\
& \quad \int_{0}^{z_{0}} d z \frac{(1+z)}{4 \pi D_{L}^{2}} \frac{d V}{d z} \int_{\tilde{L}_{1}}^{\tilde{L}_{2}} f[E(1+z)] \tilde{L} \phi(\tilde{L}, z) d \tilde{L}
\end{aligned}
$$

where $D_{L}$ is the luminosity distance, $V$ is the comoving volume, $\tilde{L}$ is the source luminosity in the energy band $x$ and $\phi$ is the luminosity function in the same band. $f[E]$ is the source spectrum at energy $E$ normalized to have unit luminosity in the band $x$. Integrating on $E$ to find the total surface brightness in band $x$ one finds

$I_{x}=\frac{c}{4 \pi} \int_{0}^{z_{\max }} d z \frac{1}{(1+z)} \frac{d t}{d z} \int_{\tilde{L}_{1}}^{\tilde{L}_{2}} \tilde{L} \phi(\tilde{L}, z) d \tilde{L}$

Applying the bolometric correction $\left(L=f_{b o l, x} \tilde{L}\right.$ with $\phi(\tilde{L}, z) d \tilde{L}=\phi(L, z) d L)$, the obscuration correction $\left(f_{\text {obsc }}\right)$ and comparing with Eq. 18 one finds that

$(1+\langle z\rangle) \frac{4 \pi I_{T}}{c}=(1+\langle z\rangle) U_{T}^{\star}=U_{T}$

where $I_{T}=f_{b o l, x} f_{o b s c} I_{x}$ and $U_{T}$ is the total comoving (as opposed to observed) energy density. The average redshift $\langle z\rangle$ is then

$(1+\langle z\rangle)=\frac{\int_{0}^{z_{\max }} d z \frac{d t}{d z} \int_{L_{1}}^{L_{2}} L \phi(L, z) d L}{\int_{0}^{z_{\max }} d z \frac{1}{(1+z)} \frac{d t}{d z} \int_{L_{1}}^{L_{2}} L \phi(L, z) d L}$

Using the luminosity function by Ueda et al. (2003) one finds $\langle z\rangle=1.1$, lower than the value $\langle z\rangle \sim 2$ assumed by previous authors. For comparison, the high luminosity objects $\left(L>10^{12} \mathrm{~L}_{\odot}\right)$ in the Ueda et al. (2003) luminosity function have $\langle z\rangle=1.3$ while the Mivaii. Hasinger. \& Schmidt (2000) and Bovle et al. (2000) have $\langle z\rangle=1.4$ and $\langle z\rangle=1.5$, respectively. With the $U_{T}^{\star}$ estimate by Elvis, Risaliti, \& Zamorani (2002) we get
$\rho_{\mathrm{BH}}=(4.7-10.6)\left[\frac{(1-\epsilon)}{9 \epsilon}\right] \times 10^{5} \mathrm{M}_{\odot} \mathrm{Mpc}^{-3}$

which is perfectly consistent with the estimate from the local BHMF $\rho_{\mathrm{BH}}=(3.2-6.5) \times 10^{5} \mathrm{M}_{\odot} \mathrm{Mpc}^{-3}$ without requiring efficiencies larger than the 'canonical' value $\epsilon=0.1$. This agreement has also been remarked by Fabian 2003 and Comastri 2003. The critical point is clearly the value of $\langle z\rangle$, the average redshift of X-ray sources emitting the XRB, which has been significantly reduced by Chandra and XMM surveys (e.g. Hasinger 2003; Fiore et al. 2003; Ueda et al. 2003). The minimum value of $\epsilon$, allowed for a consistency between the two estimates of $\rho_{\mathrm{BH}}$, is $\epsilon=0.07$ just slightly larger than the non-rotating $\mathrm{BH}$ case. Conversely, the maximum allowed efficiency is $\epsilon=0.27$, substantially below the maximally rotating Kerr $\mathrm{BH}$ case (see Sec. 6). It is intriguing to find that efficiencies smaller than those expected from non-rotating BH's or larger that those expected from maximally rotating Kerr BH's are excluded.

We have thus verified that the expected density of $\mathrm{BH}$ remnants inferred from the XRB is consistent with the local one without requiring efficiencies larger than the canonically adopted value $\epsilon=0.1$. We now verify if, with the obscured/unobscured ratios $R_{12}$ adopted in XRB synthesis models, it is possible to reproduce also the local BHMF. For the scope of this paper it suffices to notice that the Ueda et al. (2003) luminosity function, with the correction for the missing Compton-thick AGN's that we also adopt, is used by the same authors to successfully reproduce the XRB spectrum and source counts. Thus the agreement of the local and the relic BHMF in Fig. 6] is obtained by also meeting the constraints from the XRB which, in practice, provide an estimate of the number of AGN's missed by the luminosity function. The same comparison could also be done using the Mivaii. Hasinger, \& Schmidt (2000) luminosity function combined with the background model of Gilli. Salvati. \& Hasinger (2001). However, though that model is successful in reproducing the XRB spectrum and source counts, recent Chandra and XMM surveys have shown that the model redshift distribution is not correct (e.g. Hasinger 2003).

In summary, our analysis shows that it is possible to meet the XRB constraints both in terms of $\rho_{\mathrm{BH}}$ and of the local BHMF.

\section{ACCRETION EFFICIENCY AND EDDINGTON RATIO}

The relic BHMF derived from the Ueda et al. 2003) AGN luminosity function, corrected for the Compton-thick AGN's, provides a good match to the local BHMF and also satisfies the XRB constraints. The match is obtained for $\epsilon=0.1$ and $\lambda=1$. Here we investigate the locus in the $\lambda-\epsilon$ plane where an acceptable match of the local and relic BHMF's can be found, i.e. we determine the acceptable $\lambda$ and $\epsilon$ values.

To quantify the comparison between the local and relic BHMF's we recall that all the realizations of the BHMF's have a log-normal distribution at given $M$ and we consider the following expression: 
$k^{2}=\int \frac{\left[\log N_{L}(M)-\log N_{R}(M)\right]^{2}}{\sigma_{L}(M)^{2}+\sigma_{R}(M)^{2}} \mathrm{~d} \log M / \int \mathrm{d} \log M(30)$

where $N_{L}(M)$ and $N_{R}(M)$ are the local and relic BHMF's with their $1 \sigma$ uncertainties, $\sigma_{L}(M)$ and $\sigma_{R}(M)$. The integration is performed in the $\log \left(M / \mathrm{M}_{\odot}\right)=6-10$ range. $k^{2}$ is the average square deviation between the logarithms of the two BHMF's measured in units of the total standard deviation. If, for instance, $\log N_{L}(M)=\log N_{R}(M)+$ $n \sqrt{\sigma_{L}(M)^{2}+\sigma_{R}(M)^{2}}$, then $k^{2}=n^{2}$, i.e. the two functions differ, on average, by $n$ times the total standard deviation.

The $\epsilon$ and $\lambda$ values that corresponds to the minimum $k^{2}\left(k_{\min }^{2}=0.4^{2}\right)$ are marked by the filled square and are $\epsilon=0.08$ and $\lambda=0.5$. To have an acceptable match between the local and relic BHMF we require that $k^{2} \leqslant 1$ and this constraint identifies the region limited by the solid line in Fig. 17 For comparison, the dashed line limits the region where $k^{2}<0.7^{2}$, which corresponds to the average square deviation in the "canonical" case, $\epsilon=0.1$ and $\lambda=1$, which is marked by the cross. The allowed efficiencies include the non-rotating Schwarzschild BH $(\epsilon=0.054$, Schwarzschild 1916; Shapiro \& Teukolskv 1983) and are well below the maximally rotating Kerr BH $(\epsilon=0.42$, Kerr 1963; Shapiro \& Teukolskv 1983). The best agreement between the local and relic BHMF's is obtained for efficiencies larger than that of the non-rotating BH case suggesting that, on average, BH's should be rotating. Hughes \& Blandford (2003) found that BH's are typically spun down by mergers and this would limit the importance of mergers in the growth of BH's, in agreement with our assumption in the continuity equation.

The allowed Eddington ratios, $\lambda=L / L_{\mathrm{Edd}}$, are in the range $0.1<\lambda<1.7$ indicating that $\mathrm{BH}$ growth takes place during luminous accretion phases close to the Eddington limit. McLure \& Dunlop (2003), using a large sample of SDSS quasars, have recently estimated that the average $\lambda$ varies from 0.1 at $z \sim 0.2$ to 0.4 at $z \sim 2$. Accounting for the different bolometric corrections (they used $f_{b o l, B}=9.8$, $\sim 1.5$ times larger than the value adopted by us), $\lambda$ varies from 0.15 at $z \sim 0.2$ to 0.6 at $z \sim 2$. These values are in excellent agreement with the constraints posed on $\lambda$ in Fig. 7

The results in Fig. 7 do not imply that accreting BH's cannot have $\epsilon$ and $\lambda$ values outside the region of the best match between the local and relic BHMF's. Indeed, those limits are only for average efficiencies and Eddington ratios during phases in which Black Holes are significantly grown.

We conclude by noting that $\epsilon$ represents only the radiative efficiency. If there is significant release of mechanical energy, the true efficiency might be higher. For instance, in M87 the kinetic energy carried away by the jet is much larger than the radiated one Owen. Eilek. \& Kassim 2000) and, in general, the jets of radio loud AGN's can carry away up to half of the total power in kinetic energy (Rawlings \& Saunders 1991; Celotti. Padovani. \& Ghisellini 1997; Tavecchio et al. 2000). The release of mechanical and radiative energy is important for the feedback on the galaxy which is thought to be one of the causes behind the $M_{\mathrm{BH}}-\sigma_{\star}$ and $M_{\mathrm{BH}}-L_{\text {bul }}$ correlations (e.g. Silk \& Rees 1998; Blandford 1999; Begelman 2003; Granato et al. 2003). Taking into account mechanical energy, the expression $\epsilon /(1-\epsilon)$ should be transformed to $\epsilon_{R} /\left(1-\epsilon_{R}-\epsilon_{M}\right)$, where $\epsilon_{R}$ and

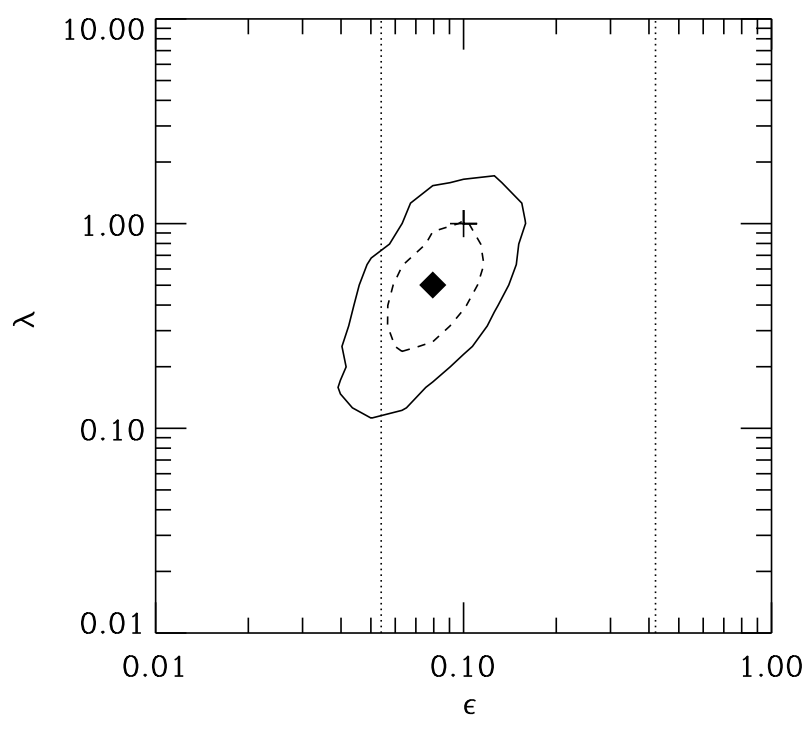

Figure 7. Locus where accretion efficiency $\epsilon$ and Eddington ratio $\lambda$ provide the best match between the local and relic BHMF's. The solid and dashed lines limit the regions where the logarithms of the local and relic BHMF differ, on average, by less than 1 and $0.7 \sigma$ (see text). The filled diamond marks the $k^{2}$ minimum and corresponds to $\epsilon=0.08$ and $\lambda=0.5$. The cross indicates $\epsilon=0.1$ and $\lambda=1$. The dashed lines are theoretical efficiencies for non-rotating $(\epsilon=0.054)$ and maximally rotating BH's $(\epsilon=0.42)$.

$\epsilon_{M}$ are the radiative and mechanical efficiencies. Then, similarly, to what has been done in section, one could place constraints on both $\epsilon_{R}$ and $\epsilon_{M}$ but this is beyond the scope of this paper.

\section{GROWTH AND ACCRETION HISTORY OF MASSIVE BLACK HOLES}

Having established that the obscuration corrected AGN luminosity function by Ueda et al. (2003) with $\epsilon=0.1$ and $\lambda=1$ provides a relic BHMF which is fully consistent with the local BHMF and the X-ray background, we can analyze the growth history of massive BH's and, in the next section, the average lifetime of their active phases.

The redshift dependence of the total density in massive BH's is given by (Eqs. [17 and 18]

$\rho_{\mathrm{BH}}\left(z^{\prime}\right)=\frac{1-\epsilon}{\epsilon c^{2}} \int_{z^{\prime}}^{z_{s}} d z \frac{d t}{d z} \int_{L_{1}}^{L_{2}} L \phi(L, z) d \log L$

and is plotted in Fig. 87. The relics of the AGN's traced with the luminosity functions by Bovle et al. (2000) and Mivaii. Hasinger. \& Schmidt (2000) reach $50 \%$ of the $z=0$ mass density around $z \sim 1.7$, while the AGN's traced by the Ueda et al. (2003) luminosity function do so at $z \sim$ 1.4. This is a consequence of the larger number of low luminosity AGN's which are present at $z \sim 1$ (see also Fig. 4b). Indeed, when separating the contributions from low $\left(M_{\mathrm{BH}}<10^{8} \mathrm{M}_{\odot}\right)$ and high mass BH's which contribute $50 \%$ each of the $z=0 \mathrm{BH}$ density, it is clear that low mass BH's grow later than high mass BH's. These, like in 

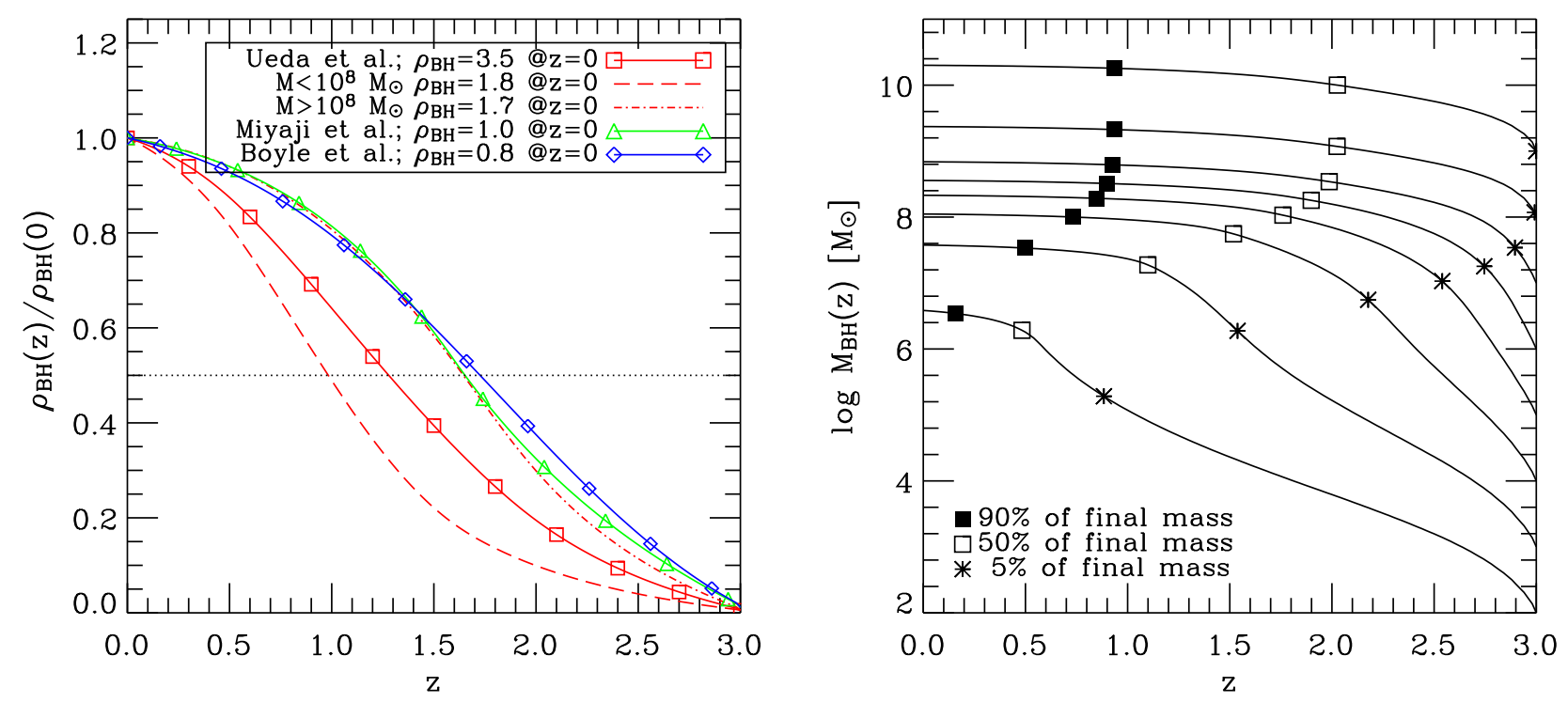

Figure 8. (a) Growth history of total BH mass density (with $\epsilon=0.1$ and $\lambda=1$ ). Different symbols indicate the use of different AGN luminosity functions. The dashed and dot-dashed lines indicate the growth history of $\rho_{\mathrm{BH}}$ in low $\left(M<10^{8} \mathrm{M}_{\odot}\right)$ and high mass $\mathrm{BH}$ 's, computed using the Ueda et al. (2003) luminosity function. The densities are normalized to the final value at $z=0$ which is reported in the upper part of the plot. The units for $\rho_{\mathrm{BH}}$ are $10^{5} \mathrm{M}_{\odot} \mathrm{Mpc}^{-3}$. (b) Average growth history of BH's with given starting mass at $z_{s}=3$ computed using the Ueda et al. (2003) luminosity function and $\epsilon=0.1, \lambda=1$. The symbols, filled squares, empty squares , and stars, indicate when the $\mathrm{BH}$ reaches $90 \%, 50 \%$, and $5 \%$ of its final mass, respectively.

the case of the AGN's traced by Bovle et al. (2000) and Mivaii. Hasinger. \& Schmidt (2000), reach $50 \%$ of their final mass at $z \sim 1.7$.

This issue can be further investigated by computing the average growth history of a $\mathrm{BH}$ with given starting, or final, mass. The growth history of a $\mathrm{BH}$ with given starting mass $M_{\mathrm{BH} 0}$ at $z_{s}$ can be estimated as follows. The average accretion rate at $z$ (or $t$ ) is given from Eq. 15 as:

$$
\langle\dot{M}(M, t)\rangle=\frac{1}{t_{E} \ln 10} \frac{(1-\epsilon) \lambda}{\epsilon N(M, t)}[\phi(L, t)]_{L=\lambda \frac{M c^{2}}{t_{E}}}
$$

thus one can solve the following differential equation to obtain the 'average' growth history of BH's:

$d M=\langle\dot{M}(M, t)\rangle \frac{d t}{d z} d z$

In Fig. 80 we plot the average growth history of BH's with different masses at $z_{s}=3$. A supermassive $\mathrm{BH}$ like that of M87 or Cygnus A $\left(M_{\mathrm{BH}} \sim 3 \times 10^{9} \mathrm{M}_{\odot}\right.$; Marconi et al. 1997; Tadhunter et al. 2003) was already quite massive ( $\left.10^{8} \mathrm{M}_{\odot}\right)$ at $z_{s}$ while a smaller $\mathrm{BH}$ like that of Centaurus A (Marconi et al. 2001) was less massive, around $M_{\mathrm{BH}}=$ $10^{6} \mathrm{M}_{\odot}$. A supermassive BH with $M_{\mathrm{BH}} \sim 10^{9} \mathrm{M}_{\odot}$ at $z_{s}=3$ should now be over $10^{10} \mathrm{M}_{\odot}$. Indeed, the existence of very massive BH's at high redshifts is suggested by the detection of very luminous quasars and, in particular, those detected at $z \sim 6$ by the SDSS survey (e.g. Fan 2003). For instance, $M_{\mathrm{BH}}$ for the farther quasar known $(z \sim 6)$ is estimated as $M_{\mathrm{BH}}=3 \times 10^{9} \mathrm{M}_{\odot}$ (Willott. McLure. \& Jarvis 2003), and one would expect its local counterpart to be more massive than $10^{10} \mathrm{M}_{\odot}$. However, these quasars have not been detected yet and it is not clear if this is because these hyper- massive BH's are very rare or they simply do not exist. In the latter case there should be a physical reason which prevents a $\mathrm{BH}$ from growing beyond $10^{10} \mathrm{M}_{\odot}$, possibly the feedback on the host galaxy mentioned in the previous sections (see also Netzer 2003).

From Fig. 8] we can also infer a confirmation of what already found in Fig. 87, namely that more massive BH's grow earlier. The symbols in the figure, filled squares, empty squares, and stars, mark the points when a BH reaches $90 \%$, $50 \%$, and $5 \%$ of its $z=0$ mass, respectively. It is clear that for $z<z_{s}=3$ all BH's gain more than $95 \%$ of their final mass but BH's which are now more massive than $10^{8} \mathrm{M}_{\odot}$ had already gained $50 \%$ of their $z=0$ mass at $z \sim 2$. Conversely, BH's which have now masses around $M_{\mathrm{BH}} \sim 10^{7} \mathrm{M}_{\odot}$ grew very recently, at $z<1$. Again, this is a consequence of the luminosity function by Ueda et al. (2003) in which the distribution of lower luminosity AGN's peaks at $z \sim 1$. Thus, the luminosity function of Ueda et al. (2003) points toward an anti-hierarchical growth of BH's in the sense that the largest $\mathrm{BH}$ 's were formed earlier.

If the correlations between $M_{\mathrm{BH}}$ and host galaxy properties were valid at higher redshifts (as is suggested by the results of Shields et al. 2003) this would immediately imply that also the most massive galaxies should form earlier, in contrast with the predictions of current semianalytic models of galaxy formation (see the introduction of Granato et al. (2003) for more details and references). The detection of high mass galaxies in sub-mm surveys is indeed more consistent with the 'monolithic' scenario in which massive ellipticals form at relatively high redshifts (e.g. Genzel et al. 2003; Granato et al. 2003 and references therein). 


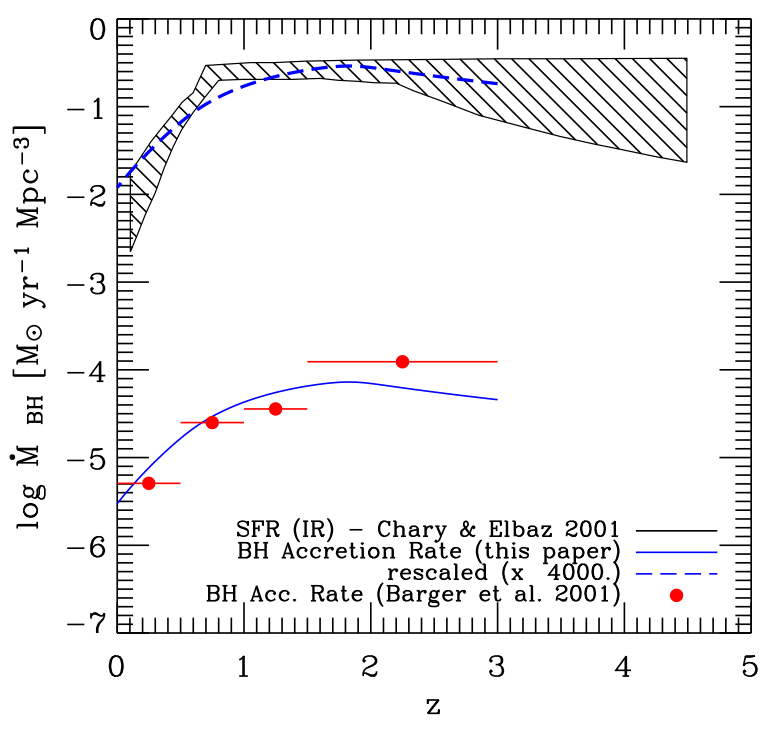

Figure 9. Cosmic BH accretion history compared with the estimate by Barger et al. (2001) and with the cosmic star formation rate by Charv \& Elbaz (2001).

The $\mathrm{BH}$ accretion history (i.e. the total accretion rate at given $z$ per unit comoving volume) can be estimated using Eq. 14 as

$\dot{M}_{A G N}(z)=\int_{M_{1}}^{M_{2}} N[M, t(z)]\langle\dot{M}[M, t(z)]\rangle d M$

In Fig. 9 we plot the cosmic accretion history of BH's (computed using the Ueda et al. (2003) luminosity function, $\epsilon=0.1$ and $\lambda=1$ ) and we compare it with the estimate by Barger et al. (2001) and with the cosmic star formation rate by Charv \& Elbaz (2001).

Our estimate of the cosmic accretion rate onto BH's agrees well with that by Barger et al. (2001), likely because most of the high redshift AGN's used by Ueda et al. (2003) come from the Barger et al. (2001) sample. Differently from us, Barger et al. (2001) estimate the bolometric AGN luminosities by integrating the observed spectral energy distributions. Thus the agreement with our analysis should be viewed as a consistency check on the bolometric corrections and on the corrections for obscured AGN's that we applied.

The cosmic accretion history has a similar redshift dependence as the cosmic star formation rate, which we report here in the form estimated by Charv \& Elbaz (2001). To aid the eye, the dashed line represents the estimate of the cosmic accretion history rescaled by 4000 . The comparison suggests that indeed the two rates have a similar redshift dependence and justifies the assumption that the accretion onto a $\mathrm{BH}$ is proportional to the star formation rate, at least at a cosmic level. This fundamental assumption is made in several models of coeval evolution of $\mathrm{BH}$ and galaxy and, together, with the feedback from the AGN explains the observed correlations $M_{\mathrm{BH}}-\sigma_{\star}$ and $M_{\mathrm{BH}}-L_{\mathrm{bul}}$ (e.g. Granato et al. 2003; Haiman. Ciotti, \& Ostriker 2003).

\section{THE LIFETIME OF ACTIVE BH'S}

We now estimate the average lifetime of active BH's with the formalism used in this paper and the corrected AGN luminosity function by Ueda et al. (2003).

In Fig. 10, we plot the BH duty cycle $\delta(M, t)$ at selected redshifts derived from Eq. [12] and computed using the Ueda et al. (2003) luminosity function, $\epsilon=0.1$ and $\lambda=1$. From Sec. 3.1 the duty cycle $\delta(M, t)$ is the fraction of BH's with mass $M$ active at time $t$ or redshift $z$. According to the definitions used in this paper, we consider a $\mathrm{BH}$ active if it is emitting at the adopted fraction $\lambda$ of the Eddington luminosity. Hence, objects which are usually classified as 'active' but which are emitting well below their Eddington limit (e.g. M87 or Centaurus A), should not be counted among the active BH's whose fraction is given by the duty cycle. BH's more massive than $\sim 10^{9} \mathrm{M}_{\odot}$ are very rarely active in the local universe (only 1 out of 10000) while they become more numerous at higher redshifts (by a factor $\sim 100$ at $z=2$ ). Conversely, lower mass BH's are usually a factor 10 more numerous. A unit duty cycle at $z=3$ is the initial condition assumed for the solution of the continuity equation (see Secs. 3.1 and 3.4). The values of the duty cycle we obtain at $z \sim 0-1$ are consistent with the average values of $3-6 \times 10^{-3}$ estimated by Haiman. Ciotti. \& Ostriker (2003).

The average duration of the accretion process, i.e. the mean lifetime of an AGN which has left a relic of mass $M$, is estimated by first solving Eq. 33 to obtain $M\left(z, M_{0}\right)$, the growth history of a BH with mass $M_{0}$ at $z_{s}$. Then the 'active' time is simply given by

$\tau(M)=\int_{0}^{z_{s}} \delta\left[M\left(z, M_{0}\right), z\right] \frac{d t}{d z} d z$

In Fig. 10p we plot the average mean lifetime of AGN's (solid line) as a function of the relic BH mass at $z=0$ computed using the Ueda et al. (2003) AGN luminosity function and $\epsilon=0.1, \lambda=1$. The average lifetime of AGN's which leave a relic $\mathrm{BH}$ mass of $>10^{9} \mathrm{M}_{\odot}$ is of the order of $1.5 \times 10^{8} \mathrm{yr}$, while for smaller relic masses $\left(<10^{8} \mathrm{M}_{\odot}\right)$ longer active phases are needed $\left(\tau_{B H} \sim 4.5 \times 10^{8} \mathrm{yr}\right)$. Considering two limiting cases from Fig. $7(\epsilon, \lambda)=(0.04,0.1)$, and $(0.15,1.6)$, the average lifetimes can increase up to $10^{9} \mathrm{yr}$. We remark that these numbers are the average lifetimes for $z<z_{s}$ with $z_{s}=3$.

The derived lifetimes are consistent with the main result from this paper that most of the BH masses are assembled via mass accretion. Indeed, if a $\mathrm{BH}$ grows by accreting matter with efficiency $\epsilon$ and emitting at a fraction $\lambda$ of its Eddington luminosity, its e-folding time is given by $t_{s}$, the Salpeter time (Salpeter 1964),

$t_{\text {Salpeter }}=\frac{\epsilon t_{E}}{(1-\epsilon) \lambda}=4.2 \times 10^{7} \mathrm{yr}\left[\frac{(1-\epsilon)}{9 \epsilon}\right]^{-1} \lambda^{-1}$

Thus, the initial BH mass has been e-folded $\sim 3$ times from $z_{s}=3$ for $M_{\mathrm{BH}}>10^{9} \mathrm{M}_{\odot}$ and more than 10 times for $M_{\mathrm{BH}}<10^{8} \mathrm{M}_{\odot}$. The apparently long lifetimes are thus the natural consequence of the fact that to grow a $\mathrm{BH}$ from small $\left(\sim 1-10 \mathrm{M}_{\odot}\right)$ or intermediate mass seeds $\left(100-1000 \mathrm{M}_{\odot}\right.$, e.g. Schneider et al. 2002) several e-folding times must pass easily implying $\tau_{B H}>10^{8} \mathrm{yr}$. For instance, to grow a $\mathrm{BH}$ from $10^{3} \mathrm{M}_{\odot}$ to $10^{9} \mathrm{M}_{\odot}$ with $\lambda=1$ and $\epsilon=0.1$, one would 

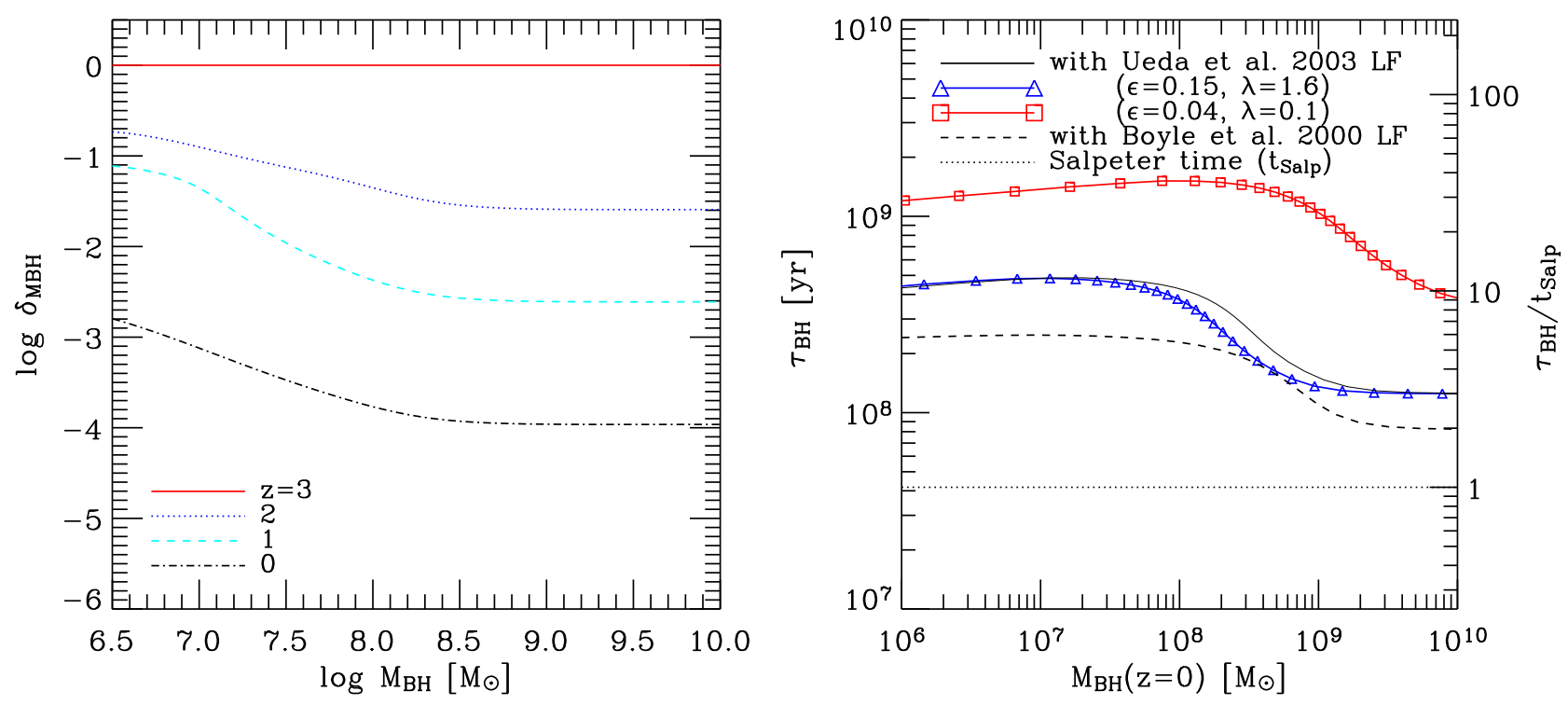

Figure 10. (a) BH duty cycle $\delta(M, z)$ at given redshifts computed with the Ueda et al. (2003) luminosity function and $\epsilon=0.1, \lambda=1$. (b) Average mean lifetime of active BH's (AGN's) as a function of the relic BH mass at $z=0$ computed with $\epsilon=0.1, \lambda=1$, the Ueda et al. (2003) (solid line) and Bovle et al. (2000) (dashed line) luminosity function. The dashed line is the corresponding Salpeter time. The scale of the $y$ axis on the right is the average lifetime in units of the Salpeter time. The lines with the empty squares and triangles are the average lifetimes computed from the Ueda et al. (2003) luminosity function with $\epsilon=0.04, \lambda=0.1$, and $\epsilon=0.15$, $\lambda=1.6$, respectively.

need $\sim 14 t_{s}$ i.e. $\sim 6 \times 10^{8} \mathrm{yr}$ (see also Haiman \& Loeb 2001). Indeed, in the model by Granato et al. (2003) of coeval evolution of $\mathrm{BH}$ and galaxy, the time needed to grow most of the $\mathrm{BH}$ mass is $\approx 3 \times 10^{8} \mathrm{yr}$ (note however that they use $\epsilon=0.15, \lambda=3$ with which the Salpeter time is roughly halved with respect to our paper).

The reason why the growth of smaller BH's $\left(<10^{8} \mathrm{M}_{\odot}\right)$ requires longer time is not obvious but can be understood from Fig. 8 8 . Consider, for instance, BH's with masses $10^{7} \mathrm{M}_{\odot}$ and $\sim 10^{10} \mathrm{M}_{\odot}$ at $z=0$. Their average growth history is traced by the lower and upper tracks in the figure. The larger $\mathrm{BH}$ has $M_{\mathrm{BH}} \sim 10^{9} \mathrm{M}_{\odot}$ at $z=3$ and has to e-fold its mass by 3 times. Conversely, the smaller $\mathrm{BH}$ has $M_{\mathrm{BH}} \sim 10^{2} \mathrm{M}_{\odot}$ at $z=3$ and has to e-fold its mass by at least ten times. The masses of large BH's $\left(>10^{8} \mathrm{M}_{\odot}\right)$ increase by smaller factors from their $z=3$ values, compared to the masses of smaller BH's. Thus they require shorter active phases.

In general, literature estimates of AGN lifetimes range from $10^{6}$ to $10^{8} \mathrm{yr}$ and are still much uncertain (see the review by Martini 2003). Models where BH's grow by a combination of gas accretion traced by short-lived $\left(\sim 10^{7} \mathrm{yr}\right)$ QSO activity and merging in hierarchically merging galaxies are consistent with a wide range of observations in the redshift range $0<z<5$ (Haehnelt 2003). However it is not surprising to find a discrepancy with our analysis since we do not consider merging and the $\mathrm{BH}$ growth history that we find is anti-hierarchical. Our estimates agree better with models in which BH's are mainly grown by gas accretion (e.g. Haiman. Ciotti. \& Ostriker 2003; Granato et al. 2003).

With an estimate of AGN lifetime based on BH demographics similar to the one presented here Yu \& Tremaine
(2002) find $\tau=0.3-1.3 \times 10^{8}$ yr for the luminous quasars using the AGN luminosity function by Bovle et al. (2000). The main reason our estimate is larger is that the Ueda et al. (2003) luminosity function has a larger number density of objects, which results in longer lifetimes. Indeed, the quasar lifetimes computed using the luminosity function by Bovle et al. (2000) are $\sim 8 \times 10^{7}$ yr (dashed line in Fig. 10b), in agreement with Yu \& Tremaine (2002).

Comparing to observational-based estimates, the lifetimes from this paper are in agreement with the results from the length of radio jets (see Martini 2003 for more details). More recently, Miller et al. (2003) found from SDSS data that a very high fraction of galaxies host an AGN $(\sim 20-40 \%)$ suggesting lifetimes longer than previously thought (i.e. $>10^{8} \mathrm{yr}$ ). Finally, our estimate is in agreement with the upper limit of $10^{9} \mathrm{yr}$ set by the timescale over which the quasar luminosity density rises and falls (see, e.g., Osmer 2003).

In summary, we estimate that local high mass BH's $\left(M>10^{9} \mathrm{M}_{\odot}\right)$ have been active, on average, $\sim 1.5 \times 10^{8} \mathrm{yr}$. On the contrary, the assembly of lower mass BH's has required active phases lasting at least three times that much $\left(\sim 4.5 \times 10^{8} \mathrm{yr}\right)$. These average lifetimes can be as large as $\sim 10^{9}$ if one considers the smaller efficiency and fraction of Eddington luminosity which are still compatible with local BH's $(\epsilon=0.04, \lambda=0.1)$.

\section{CONCLUSIONS}

We have quantified the importance of mass accretion during AGN phases in the growth of supermassive black holes 
(BH) by comparing the mass function of black holes in the local universe with that expected from AGN relics, which are black holes grown entirely during AGN phases.

The local BH mass function (BHMF) has been estimated by applying the well-known correlations between $\mathrm{BH}$ mass, bulge luminosity and stellar velocity dispersion to galaxy luminosity and velocity functions. We have found that different BH-galaxy correlations provide the same BHMF only if they have the same intrinsic dispersion, confirming the findings of Marconi \& Hunt (2003). The density of supermassive black holes in the local universe is $\rho_{\mathrm{BH}}=4.6_{-1.4}^{+1.9} h_{0.7}^{2} \times 10^{5} \mathrm{M}_{\odot} \mathrm{Mpc}^{-3}$.

The relic BHMF is derived from the continuity equation with the only assumption that AGN activity is due to accretion onto massive BH's and that merging is not important. We find that the relic BHMF at $z=0$ is generated mainly at $z<3$ where the major part of BH's growth takes place. The relic BHMF at $z=0$ is very little dependent on its value at $z_{s}=3$ since the main growth of BH's took place at $z<3$. Moreover, the $\mathrm{BH}$ growth is anti-hierarchical in the sense that smaller BH's $\left(M_{B H}<10^{8} M_{\odot}\right)$ grow at lower redshifts $(z<1)$ with respect to more massive one's $(z \sim 1-3)$. If the correlations between BH mass and hostgalaxy-properties hold at higher redshifts this would represent a potential problem for hierarchical models of galaxy formation.

Unlike previous work, we find that the BHMF of AGN relics is perfectly consistent with the local BHMF indicating the local black holes were mainly grown during AGN activity. This agreement is obtained while satisfying, at the same time, the constraints imposed from the X-ray background both in terms of $\mathrm{BH}$ mass density and fraction of obscured AGN's. The reasons for the solution of the discrepancy at high masses found by other authors are the following:

- we have taken into account the intrinsic dispersion of the $M_{\mathrm{BH}}-\sigma_{\star}$ and $M_{\mathrm{BH}}-L_{\mathrm{bul}}$ correlations in the determination of the local BHMF;

- we have adopted the coefficients of the $M_{\mathrm{BH}}$ $\sigma_{\star}$ and $M_{\mathrm{BH}}-L_{\mathrm{bul}}$ ( $K$ band) correlations derived by Marconi \& Hunt (2003) after considering only 'secure' BH masses;

- we have derived improved bolometric corrections which do not take into account reprocessed IR emission in the estimate of the bolometric luminosity.

The comparison between the local and relic BHMF's also suggests that the merging process at low redshifts $(z<3)$ is not important in shaping the relic BHMF, and allows us to estimate the average radiative efficiency $(\varepsilon)$, the ratio between emitted and Eddington luminosity $(\lambda)$ and the average lifetime of active BH's.

Our analysis thus suggests the following scenario: local black holes grew during AGN phases in which accreting matter was converted into radiation with efficiencies $\varepsilon=0.04-0.16$ and emitted at a fraction $\lambda=0.1-1.7$ of the Eddington luminosity. The average total lifetime of these active phases ranges from $\simeq 4.5 \times 10^{8} \mathrm{yr}$ for $M_{B H}<10^{8} M_{\odot}$ to $\simeq 1.5 \times 10^{8}$ yr for $M_{B H}>10^{9} M_{\odot}$ but can become as large as $\sim 10^{9}$ yr for the lowest acceptable $\epsilon$ and $\lambda$ values.

\section{ACKNOWLEDGMENTS}

A.M. and L.K.H. acknowledge support by MIUR (Cofin0102-02). A.M., G.R., and R.M., acknowledge support by INAOE, Mexico, during the 2003 Guillermo-Haro Workshop where part of this work was performed. This research has made use of NASA's Astrophysics Data System Bibliographic Services.

\section{REFERENCES}

Adams F. C., Graff D. S., Mbonye M., Richstone D. O., 2003, ApJ, 591, 125

Aller M. C., Richstone D., 2002, AJ, 124, 3035

Antonucci R., 1993, ARA\&A, 31, 473

Barger A. J., Cowie L. L., Bautz M. W., Brandt W. N., Garmire G. P., Hornschemeier A. E., Ivison R. J., Owen F. N., 2001, AJ, 122, 2177

Barth A. J., Sarzi M., Rix H., Ho L. C., Filippenko A. V., Sargent W. L. W., 2001, ApJ, 555, 685

Begelman M. C., 2003, Carnegie Observatories Astrophysics Series, Vol. 1: Coevolution of Black Holes and Galaxies, ed. L. C. Ho (Pasadena: Carnegie Observato$\operatorname{ries}^{1}$ )

Bernardi M. et al., 2003, AJ, 125, 1817

Bernardi M. et al., 2003, AJ, 125, 1849

Blandford R. D., 1999, in Galaxy Dynamics, eds, D, R. Merritt, M. Valluri, \& J. A. Sellwood (San Francisco: ASP), 87

Boyle B. J., Shanks T., Croom S. M., Smith R. J., Miller L., Loaring N., Heymans C., 2000, MNRAS, 317, 1014

Cattaneo A., Haehnelt M. G., Rees M. J., 1999, MNRAS, 308, 77

Cavaliere A., Vittorini V., 2002, ApJ, 570, 114

Cavaliere A., Padovani P., 1989, ApJ, 340, L5

Cavaliere A., Morrison P., Wood K., 1971, ApJ, 170, 223

Celotti A., Padovani P., Ghisellini G., 1997, MNRAS, 286, 415

Chary R., Elbaz D., 2001, ApJ, 556, 562

Chokshi A., Turner E. L., 1992, MNRAS, 259, 421

Ciotti L., van Albada T. S., 2001, ApJ, 552, L13

Cole S. et al., 2001, MNRAS, 326, 255

Comastri A., 2003, AIP Conf. Proc., The Astrophysics of Gravitational Wave Sources, ed. J. Centrella, in press (astro-ph/0307426)

Comastri A., Setti G., Zamorani G., Hasinger G., 1995, A\&A, 296, 1

Di Matteo T., Croft R. A. C., Springel V., Hernquist L., 2003, ApJ, 593, 56

Elvis M., Risaliti G., Zamorani G., 2002, ApJ, 565, L75

Elvis M. et al., 1994, ApJS, 95, 1

Erwin P., Graham A. W., Caon N., 2003, Carnegie Observatories Astrophysics Series, Vol. 1: Coevolution of Black Holes and Galaxies, ed. L. C. Ho (Pasadena: Carnegie Observatories $^{1}$ )

Fabian A. C., 2003, Carnegie Observatories Astrophysics Series, Vol. 1: Coevolution of Black Holes and Galaxies, ed. L. C. Ho (Pasadena: Carnegie Observatories ${ }^{1}$ )

Fabian A. C., Iwasawa K., 1999, MNRAS, 303, L34 
Fan X., 2003, Carnegie Observatories Astrophysics Series, Vol. 1: Coevolution of Black Holes and Galaxies, ed. L. C. Ho (Pasadena: Carnegie Observatories ${ }^{1}$ )

Ferrarese L., 2002, in "Current High-Energy Emission around Black Holes", Eds. C.-H. Lee \& H.-Y. Chang (Singapore: World Scientific), p. 3

Ferrarese L., Merritt D., 2000, ApJ, 539, L9

Fiore F. et al., 2003, A\&A, 409, 79

Fukugita M., Hogan C. J., Peebles P. J. E., 1998, ApJ, 503, 518

Fukugita M., Shimasaku K., Ichikawa T., 1995, PASP, 107, 945

Gebhardt K. et al., 2000, ApJ, 539, L13

Genzel R., Baker A. J., Tacconi L. J., Lutz D., Cox P., Guilloteau S., Omont A., 2003, ApJ, 584, 633

George I. M., Turner T. J., Netzer H., Nandra K., Mushotzky R. F., Yaqoob T., 1998, ApJS, 114, 73

Gilli R., Salvati M., Hasinger G., 2001, A\&A, 366, 407

Gilli R., Risaliti G., Salvati M., 1999, A\&A, 347, 424

Gonzalez A. H., Williams K. A., Bullock J. S., Kolatt T. S., Primack J. R., 2000, ApJ, 528, 145

Granato G. L., De Zotti G., Silva L., Bressan A., Danese L., 2003, ApJ, in press (astro-ph/0307202

Haehnelt M. G., 2003, Carnegie Observatories Astrophysics Series, Vol. 1: Coevolution of Black Holes and Galaxies, ed. L. C. Ho (Pasadena: Carnegie Observatories ${ }^{1}$ )

Haehnelt M. G., Kauffmann G., 2000, MNRAS, 318, L35

Haehnelt M. G., Natarajan P., Rees M. J., 1998, MNRAS, 300,817

Haiman Z., Ciotti L., Ostriker J. P., 2003, ApJ, submitted (astro-ph/0304129)

Haiman Z., Loeb A., 2001, ApJ, 552, 459

Hasinger G., 2003, AIP Conf. Proc., The Emergence of Cosmic Structure: Thirteenth Astrophysics Conference, S. S.

Holt \& C. Reynolds eds., 666, 227

Hatziminaoglou E., Mathez G., Solanes J., Manrique A., Salvador-Solé E., 2003, MNRAS, 343, 692

Hughes S. A., Blandford R. D., 2003, ApJ, 585, L101

Hunt L. K., Pierini D., Giovanardi C., 2003, A\&A, in press (astro-ph/0311213)

Kauffmann G., Haehnelt M., 2000, MNRAS, 311, 576

Kerr R. P., 1963, Phys. Rev. Lett., 11, 237

Kochanek C. S. et al., 2001, ApJ, 560, 566

Kormendy J., Gebhardt K., 2001, in 20th Texas Symposium on Relativistic Astrophysics, eds. J. C. Wheeler and H. Martel (Melville, NY: AIP), 363

Kormendy J., Richstone D., 1995, ARA\&A, 33, 581

Lynden-Bell D., 1969, Natur, 223, 690

Macchetto F., Marconi A., Axon D. J., Capetti A., Sparks

W., Crane P., 1997, ApJ, 489, 579

Magdziarz P., Zdziarski A. A., 1995, MNRAS, 273, 837

Magorrian J. et al., 1998, AJ, 115, 2285

Maiolino R., Rieke G. H., 1995, ApJ, 454, 95

Marconi A., Hunt L. K., 2003, ApJ, 589, L21

Marconi A., Salvati M., 2002, in Issues in Unification of Active Galactic Nuclei, eds. R. Maiolino, A. Marconi and N. Nagar (San Francisco: ASP), 217

Marconi A., Capetti A., Axon D. J., Koekemoer A., Macchetto D., Schreier E. J., 2001, ApJ, 549, 915

Marconi A., Axon D. J., Macchetto F. D., Capetti A., Soarks W. B., Crane P., 1997, MNRAS, 289, L21

Martini P., 2003, Carnegie Observatories Astrophysics Se- ries, Vol. 1: Coevolution of Black Holes and Galaxies, ed. L. C. Ho (Pasadena: Carnegie Observatories ${ }^{1}$ )

Marzke R. O., Geller M. J., Huchra J. P., Corwin H. G., 1994, AJ, 108, 437

McLure R. J., Dunlop J. S., 2003, MNRAS submitted (astro-ph/0310267)

McLure R. J., Dunlop J. S., 2002, MNRAS, 331, 795

Menci N., Cavaliere A., Fontana A., Giallongo E., Poli F., Vittorini V., 2003, ApJ, 587, L63

Merritt D., Ferrarese L., 2001, in The Central Kiloparsec of Starbursts and AGN, eds. J. H. Knapen et al. (San Francisco: ASP), 335

Merritt D., Ferrarese L., 2001, MNRAS, 320, L30

Miller C. J., Nichol R. C., Gomez P., Hopkins A., Bernardi M., 2003, ApJ, in press (astro-ph/0307124)

Miyaji T., Hasinger G., Schmidt M., 2000, A\&A, 353, 25

Monaco P., Salucci P., Danese L., 2000, MNRAS, 311, 279

Nakamura O., Fukugita M., Yasuda N., Loveday J., Brinkmann J., Schneider D. P., Shimasaku K., SubbaRao M., 2003, AJ, 125, 1682

Netzer H., 2003, ApJ, 583, L5

Nulsen P. E. J., Fabian A. C., 2000, MNRAS, 311, 346

Osmer P. S., 2003, Carnegie Observatories Astrophysics Series, Vol. 1: Coevolution of Black Holes and Galaxies, ed. L. C. Ho (Pasadena: Carnegie Observatories ${ }^{1}$ )

Owen F. N., Eilek J. A., Kassim N. E., 2000, ApJ, 543, 611 Padovani P., Burg R., Edelson R. A., 1990, ApJ, 353, 438 Perola G. C., Matt G., Cappi M., Fiore F., Guainazzi M., Maraschi L., Petrucci P. O., Piro L., 2002, A\&A, 389, 802 Rawlings S., Saunders R., 1991, Natur, 349, 138

Risaliti G., Maiolino R., Salvati M., 1999, ApJ, 522, 157

Salpeter E. E., 1964, ApJ, 140, 796

Salucci P., Szuszkiewicz E., Monaco P., Danese L., 1999, MNRAS, 307, 637

Schmidt M., 1963, Natur, 197, 1040

Schneider R., Ferrara A., Natarajan P., Omukai K., 2002, ApJ, 571, 30

Schwarzschild K., 1916, Sitzungsber. Dtsch. Akad. Wiss. Berlin, Kl. Math. Phys. Tech., 189

Setti G., Woltjer L., 1989, A\&A, 224, L21

Shapiro S. L., Teukolsky S. A., 1983, "Black Holes, White

Dwarfs, and Neutron Stars" (New York: Wiley)

Sheth R. K. et al., 2003, ApJ, 594, 225

Shields G. A., Gebhardt K., Salviander S., Wills B. J., Xie B., Brotherton M. S., Yuan J., Dietrich M., 2003, ApJ, 583, 124

Silk J., Rees M. J., 1998, A\&A, 331, L1

Simien F., de Vaucouleurs G., 1986, ApJ, 302, 564

Small T. A., Blandford R. D., 1992, MNRAS, 259, 725

Sołtan A., 1982, MNRAS, 200, 115

Steidel C. C., Adelberger K. L., Giavalisco M., Dickinson M., Pettini M., 1999, ApJ, 519, 1

Tadhunter C., Marconi A., Axon D., Wills K., Robinson T. G., Jackson N., 2003, MNRAS, 342, 861

Tavecchio F. et al., 2000, ApJ, 543, 535

Telfer R. C., Zheng W., Kriss G. A., Davidsen A. F., 2002, ApJ, 565, 773

Tremaine S. et al., 2002, ApJ, 574, 740

Ueda Y., Akiyama M., Ohta K., Miyaji T., 2003, ApJ, in press (astro-ph/0308140)

Vanden Berk D. E. et al., 2001, AJ, 122, 549 
van der Marel R. P., de Zeeuw P., Rix H.-W., Quinlan G. D., 1997, Natur, 385, 610

Vignali C., Brandt W. N., Schneider D. P., 2003, AJ, 125, 433

Volonteri M., Haardt F., Madau P., 2003, ApJ, 582, 559

Willott C. J., McLure R. J., Jarvis M. J., 2003, ApJ, 587, L15

Wyithe J. S. B., Loeb A., 2003, ApJ, 595, 614

Yu Q., Tremaine S., 2002, MNRAS, 335, 965

Yuan W., Brinkmann W., Siebert J., Voges W., 1998, A\&A, 330, 108

Zamorani G. et al., 1981, ApJ, 245, 357

Zel'dovich Y. B., Novikov I. D., 1964, Sov. Phys. Dokl., 158,811 